\title{
Energy Allocation Design for the Satellite Return Channel of a Massive NOMA System with Interference Cancellation
}

\author{
Francesc Molina, Student Member, IEEE, Josep Sala-Álvarez, Senior Member, IEEE
}

\begin{abstract}
The energy allocation problem in a massive access setting under iterative successive interference cancellation (SIC) is undertaken in this work. The classic one-iteration SIC still has room for improvement when the channel coding schemes adopted are non-error free. In this work, we investigate a satellite return link in which transmitters employ the same physical layer and transmission protocol: a given encoder, and spreading-based nonorthogonal multiple access. Leveraging iterative SIC, the gateway is endowed with strong interference suppression potential to deal with many uncoordinated transmissions. We elaborate on the interplay between energy and reliability for all users by designing an energy allocation with minimum energy expenditure when the iterative SIC receiver performs two decoding attempts per user. We resort to the user-asymptotic regime to address the randomness in the multi-feature system model of two-iteration SIC, which has turned the analysis into a deterministic one. We formulate our final design as a vector optimization aided by tools from the calculus of variations. We show the competitive performance achieved by the designed allocation, specially under encoders associated with short-length codes. We also assess our analysis by simulating a low-level SIC implementation.
\end{abstract}

Index Terms-Massive multiple access, iterative successive interference cancellation, satellite, short packet, energy allocation.

\section{INTRODUCTION}

$\mathbf{T}$ HE upcoming massive machine-type communications context is envisaging many devices simultaneously communicating among one another or with a central node in innovative ways [1]. An explosive growth of smart devices enabling short packet transmissions is expected. Hence, the main target nowadays is the analysis of multiple access schemes that can support such a high number of possibly small and powerefficient devices, distributed either over a large area or more densely over a smaller area, and that transmit infrequently whenever they are ready or on a periodic scheduled basis if, for example, they track some continuous parameter [2].

Satellite networks constitute a potential solution to handle this kind of traffic in delay-tolerant applications [3] or to create a resilient backhaul, since they cover remote areas and enable high throughput at a reasonable cost [4]. Other advantages are the easy implementation of low-cost fare plans for small data

Work supported in part by Agencia Estatal de Investigación (AEI, Spain) and by European Regional Development Fund (ERDF) through project RODIN (PID2019-105717RB-C22), and in part by the Catalan administration (AGAUR) under Grant 2017 SGR 578 and Fellowship 2020FI_B2 00062.

The present article was partially presented at the 2020 54th IEEE Int. Conference on Communications in May 2020.

Corresponding author: Francesc Molina.

The authors are with the Department of Signal Theory and Communications, Universitat Politècnica de Catalunya, 08034 Barcelona, Spain (e-mail: francesc.molina@upc.edu; josep.sala@upc.edu). exchange and demand-based fares, resource management flexibility and cost/effectiveness in broadcast/multicast connectivity. In this context of the Internet of Remote Things (IoRT), the commonest satellite-based architectures consider direct-tosatellite connectivity (DtS) when devices have medium/high complexity (with power-efficient transceivers and directive antennas) or two-layer architectures, where the intermediate nodes operate as aggregation relay nodes, when devices are simple [5]-[8]. Given the relevance of satellite IoRT systems and their capability to offload traffic from the terrestrial infrastructure, many companies are competing to "conquer" some space orbits in favour of miniaturized satellites, equipped with simplified on-board signal processing, that operate as transponders and rely on ground stations for processing [9].

In networks comprising massive connectivity and users generating low traffic (with low transmission duty cycles and low bit-payload), the shared multiple access problem can be tackled in a number of ways, for instance, using orthogonal multiple access (OMA) techniques such as time, frequency or code-division multiple access. Nevertheless, the former techniques require stringent coordination between devices and the satellite, which may be burdensome to the satellite's link performance, specially so in the GEO orbit due to the long trip time [10]. Additionally, OMA techniques are intrinsically limited by scarce orthogonal resources, a fact that limits communication performance in presence of many users. The most consensual scheme to these two problems is thus to enable many simultaneous transmissions by using non-orthogonal multiple access (NOMA) [11]. Typically, users share the same time and frequency band, and the code or power domain is exploited by a successive interference cancellation (SIC) receiver.

A number of solutions to enhance the performance of random access (RA) IoRT-oriented satellite networks have been applied together in the latest years [12], specially so, for the satellite return link. Such is the case for Slotted ALOHA (SA) and its first successor in incorporating temporal diversity, Diversity-Framed SA (DFSA), which is, finally, also endowed with interference cancellation in Contention Resolution Diversity SA (CRDSA) [13]. A competitive NOMA scheme for RA satellite return links with even less coordination consists of users using the same encoder and increasing their transmission time leveraging spreading signatures on a per-symbol basis of dimension $N$ to separate users [14]. Conceptually, the idea is to work in a higher dimensional signal space where users interfere with each other only in some dimensions. A high number of users $K$ is supported, for which, in absence of coordination among signatures of users, power is allocated uniformly in each dimension. This allows for a deterministic 
analysis of RA satellite systems as a function of the number of users per dimension $K / N$ [15], [16], and for powerful interference mitigation thanks to the low cross-correlation between spreading signatures. This strategy, in combination with SIC after the bank of single-user matched filters, is precisely that adopted in Enhanced Spread Spectrum ALOHA (E-SSA); users operate uncoordinately since complexity is moved to the iterative SIC ground-based gateway, that resolves packet collisions in a highly effective way when packet-power unbalance is enabled. E-SSA's splendid performance has led to its application for machine-type terminals accessing satellites operating in the S-band [17] or in the $\mathrm{C} / \mathrm{Ku} / \mathrm{Ka}$-band [18], and also in the return channel of the ANTARES aeronautical communication system. Unveiling the relevance of SIC in the emergent IoRT [19], [20], this work elaborates precisely an original point of view on the energy allocation design, from an energy efficiency perspective, considering short packets and practical features of the iterative SIC receiver.

\section{A. Related Works and Research Contributions}

Pioneering works such as [21] have derived theoretical allocations in the infinite blocklength regime for a number of NOMA settings with SIC in which, since channel decoding is error free, all users can be decoded successfully. Nevertheless, the validity of previous analyses for short packets has been widely discussed, showing that they are inaccurate for blocklengths of hundreds of symbols used by the IoRT nowadays. Rather, results from optimal second-order coding schemes can be adopted [22]. An example of their application to capture the reliability of such theoretical coding schemes is shown in [23], [24]. A consensual procedure to evaluate the reliability of practical decoders is by computing their packet error rate (PER) characteristics; their application in SIC receivers under energy-limited settings finishes the receiver's operation with some users decoded unsuccessfully [25]-[27]. In this respect, an improved scheme to partially counteract such a performance loss is to iterate the SIC algorithm on surviving users [28]. This approach, however, is complex to model with mathematical rigor, mainly because channel decoding operates with memory of previous iterations when decodes every user. The studies carried in the literature, e.g. [28], overlook this detail for system modeling; a fact that, to the best of our knowledge, we consider incorrect in view of the findings in [29]. In this paper, we fill the gap by investigating an optimal energy allocation under an accurate model for the iterative SIC receiver.

In this regard, a number of designs have been followed, mainly, in satellite scenarios. For such designs, more attention has been paid to the statistical model for interference and to the technique used to design the optimal allocation. In [25], stochastic geometry is used to characterize the probability density function of interference when a low number of users access the satellite under FDSA. In [30], the conjoint system analysis of CRDSA with transmit power diversity plus SIC is based on a bipartite graph representation. For networks operating under E-SSA, interference turns out to be Gaussian as the large spreading factor randomizes interference after matched filtering and, if the number of users is sufficiently high, each of the SIC's decoding-cancellation operations can be modeled accurately in a simple manner [26], [27].

The allocation designs already performed consider some of the interplays between energy, rate, blocklength, and reliability when SIC operates. In [28], many users use the same forward error correction code, and a semi-analytical approach shows the competitiveness of exponentially distributed energies under an iterative SIC implementation. This result is supported by variational calculus-based findings in [21] that derive, for one-iteration SIC in the user-asymptotic regime, the optimal energy-rate allocation subject to error-free performance. The energy-reliability interplay is analyzed in [26] for a practical one-iteration SIC with the same rate and blocklength allocated to all users and the goal focused on maximizing spectral efficiency, wherein the signal-to-interference-plus-noise ratios (SINRs) of users are practically equal and the reliability of users are decreasing in the SIC decoding order. In [27], the previous analysis is repeated under a two-iteration SIC, showing a reduction in energy unbalance and a radically different behavior in terms of the SINR each user experiences. The transmit energy minimization is studied in [31] with variable blocklengths and the heuristic assumption of full interference.

In this paper, we focus on the analysis and optimization of a DtS setting under short packets and an iterative SIC receiver. Our analysis can be also applied to two-layer networks [7], [8] provided that the number of intermediate relay nodes is sufficiently large. We overcome the shortcomings of the stateof-the-art and comprehensively evaluate the performance of a massive access satellite return link in the user-asymptotic regime. We have tackled, for the same channel encoder, as in [27], [28], the most appropriate scenario of practical interest in which energy allocation minimizes energy expenditure while guaranteeing a reliability measure. The main substance and contributions of this work are condensed next:

- We design an energy allocation loaded to users, and that allows for selecting the transmitted symbol energy of each user in an uncoordinated manner based on the channel power gain accurately estimated by each user. The latter, differs from the analysis in [26], [27] which elaborate on the optimal distribution at the SIC receiver's input.

- We characterize the accurate performance of a twoiteration SIC in terms of a simple system model, rather than the approximate analysis shown in [28]. More specifically, we characterize channel decoding statistically by the univariate PER in the first SIC iteration, and we add an additional argument to construct the bivariate PER function for the second SIC iteration.

- Randomness due to the number of users and to channel decoding are addressed in the user-limit case, wherein, moreover, user-indices at each iteration admit an asymptotic relationship that is, in fact, the thread of this work.

- We explore the optimal packet-power or -energy unbalance in the user-asymptotic case by minimizing energy expenditure subject to fair or unfair allocation in terms of per-user or average network reliability. To that aim, we consider twice continuously differentiable functions, and we resort to an iterative resolution method so as to obtain the final designs in a high performance/complexity ratio. 
- We thoroughly assess the performance attained by the proposed design for a standardized error correcting code and a low-level implementation of iterative SIC.

The strength of our analysis is that it permits the evaluation of the performance attained by two-iteration SIC for both theoretical and practical coding schemes as long as their univariate PER versus SINR curves are known.

\section{B. Paper Organization and Notation}

Section II summarizes the system set-up and the operation of iterative SIC. Section III analyses the system model in terms of SINR expressions. Section IV studies the user-asymptotic behavior of the previous system model. Section V is devoted to optimization purposes. Section VI describes the numerical results, and conclusions are reported in Section VII.

Paper notation: Vectors are denoted in boldface. $\log$ is the natural logarithm. $\|\cdot\|$ is the $\ell_{2}$-norm. $\mathbb{E}[\cdot]$ is the expectation operator. $y^{\prime}(x)$ is the first derivative of $y(x)$.

\section{System Model}

We consider a return channel scenario where a satellite node is acting as a relay between a large number of users $K$ and a ground-based gateway station. Users are connected to the gateway through slowly time-varying channels whose instantaneous power gains

$$
h[1], h[2], \ldots, h[K]
$$

are perfectly known to them. This is possible, to a sufficiently high accuracy, if the above channel power gains are dominated by strong line-of-sight ${ }^{1}$, and users estimate and track their channels from a downlink pilot signal [26].

We adopt, as in relevant analyses of satellite settings [26][28], the framework in E-SSA: a common physical layer for users, consisting of the same modulation and error correcting code, and spreading-based multiple access. We respectively denote the encoding and decoding functions $f(\cdot)$ and $f^{-1}(\cdot)$. User $k$ encodes the vector $\mathbf{b}_{k}$ of $b$ bits: it applies the encoding function to generate the $n$-symbol packet $\mathbf{s}_{k}=f\left(\mathbf{b}_{k}\right)$, and sets the average symbol energy $E_{\mathrm{x}}[k] . \mathbf{s}_{k}$ consists of $n_{o}$ preamble symbols plus $n_{e}$ encoded symbols; the coding rate is $R=b / n_{e}$. Spreading-based multiple access is adopted for individual low-power consumption and to cope with low peak-to-average-power ratios, at the cost of increasing the users' transmission time. For these reasons and complying with previous works [26]-[28], users sign packets leveraging spreading codes with period much higher than the symbol interval $^{2} . c_{k, m}(\mathrm{t})$ is the spreading waveform of user $k$ for symbol $m$, which has an average per-symbol cross-correlation gain proportional to the inverse of the spreading factor $N$ [14]. Then, packets are transmitted on the satellite link.

When the number of users is sufficiently large over the coverage area and its ordered profile $h$ of channel power gains is known, we can design an allocation function $g(h)$ based on

\footnotetext{
${ }^{1}$ The multipath components in a channel dominated by strong line-ofsight contribute to increasing the system noise level after symbol despreading as the receiver only processes the line-of-sight component for each user.

${ }^{2}$ to have Gaussian distributed interference at the despreader's output.
}

this channel power gain profile. $g(h)$ is then used by user $k$ to obtain its transmitted symbol energy by just applying its accurate estimation $h[k]$ as argument of the known allocation, as $E_{\mathrm{x}}[k]=g(h[k])$. The aim of this work is the design of such an allocation for a gateway station that features a practical iterative SIC strategy, thus outperforming the classical oneiteration SIC approach.

\section{A. Satellite Operation and Gateway Demodulation}

We consider a satellite transponder with simplified on-board processing that changes the frequency of the uplink signal and transmits it to a ground-based gateway station [9], [26]. We adopt, for the satellite transponder, a traveling-wave amplifier operating in the linear zone: whereby, non-linear distortion is compensated for analogically [32], [33] and the operating point is found below saturation. At the gateway, the user-aggregate signal is received under Gaussian noise $w(\mathrm{t})$ as

$$
y(\mathrm{t})=\sum_{k=1}^{K} A_{\mathrm{r}}[k] \sum_{m=0}^{n-1} \mathbf{s}_{k}[m] c_{k, m}\left(\mathrm{t}-m T-\tau_{k}\right)+w(\mathrm{t}),
$$

where the symbol sequence for user $k$ is received with complex amplitude $A_{\mathrm{r}}[k]$ and square modulus $\left|A_{\mathrm{r}}[k]\right|^{2}=E_{\mathrm{x}}[k] h[k]$ if the channel remains stationary during the transmission time, and with delay $\tau_{k} . T$ is the symbol period.

At the gateway, SIC results a very competitive approach to process the received signal $y(\mathrm{t})$ when the signatures employed by users are known to the receiver. The majority of works in the literature have adopted the classical SIC receiver constituted by one iteration due to its amenable treatment and its satisfactory performance. However, its ideal performance when using error-free coding schemes is put in doubt when users employ coding schemes for short packets; actually, our key system design. In this case, since after processing all users, some of them may be eventually decoded unsuccessfully, the receiver can iterate the adopted SIC approach over users decoded unsuccessfully. This is possible if users compute a cyclic redundancy check (CRC) and encapsulate it with data, to allow for reliable packet error detection after channel decoding, rather than taking the soft-decision outputs as the symbol reliability measures. Hence, on the basis of [29], we adopt a SIC strategy constituted by 2 iterations, each comprising as many stages as users survive at the beginning of each iteration. We distinguish between iteration, which refers to each of the repetitions of the SIC algorithm, and stage, which brings together the steps of demodulation and decoding for a given user. We detail the signal level system model of the adopted receiver in the following subsections.

\section{B. Receiver's Operation: The First Iteration}

At the beginning of the first iteration, we rank users according to their strengths through the indexing $1 \leq k_{1} \leq K$. We assume that, as the strengths received from users depend on the designed allocation, such an ordering coincides with the order established from the channel power gains:

$$
h[1] \geq \cdots \geq h\left[k_{1}\right] \geq \cdots \geq h[K] .
$$


So, we refer indistinctly to strongest (or weakest) user with respect to the one who has better (or worst) channel conditions or received symbol energy.

With regard to SIC demodulation, the receiver performs $K$ stages. In each of them, the yet unprocessed strongest user is demodulated, assuming known its spreading waveform [16], [28], and decoded. The output of the symbol despreader for user $k_{1}$ is

$$
\mathbf{y}_{k_{1}}^{1}=A_{\mathrm{r}}\left[k_{1}\right] \mathbf{s}_{k_{1}}+\sqrt{N_{t}^{1}\left[k_{1}\right]} \mathbf{w}_{k_{1}}^{1} .
$$

For convenience, superscripts indicate the iteration index $i$, e.g. $i=1$ in this case. $\mathbf{w}_{k}^{1}$ is a unit-variance Gaussian term consisting of the thermal noise plus the waveforms associated with the interference from all previously processed and yet unprocessed users at stage $k_{1} . N_{t}^{1}\left[k_{1}\right]$ is the level of noise plus interference at stage $k_{1}$ in the first iteration. For the computation of the latter term, the cross-correlations between the $k_{1}$-th user spreading waveform and other signals $k \neq k_{1}$ are taken into account. Afterwards, channel decoding operates as $\hat{\mathbf{b}}_{k_{1}}=f^{-1}\left(\mathbf{y}_{k_{1}}^{1}\right)$ to estimate the bit stream transmitted by user $k_{1}$. Next, CRC is checked out to determine whether successful decoding occurred or not, and, only when the CRC succeeds, the $k_{1}$-th user is regenerated at the waveform level as $\hat{r}_{k_{1}}(\mathrm{t})$ and is canceled from the input signal $y(\mathrm{t})$. In this respect, assuming the timing estimation not critical if $y(\mathrm{t})$ in (2) is sampled at least twice the chip rate, the main issue affecting the reconstruction of users is the complex amplitude estimation of the detected constellation symbols. Our approach reconstructs the symbol sequence of each user as $\hat{\mathbf{r}}_{k_{1}}^{\mathrm{ok}}=\hat{A}_{\mathrm{r}}\left[k_{1}\right] \mathbf{s}_{k_{1}}$, with the complex amplitude computed by the cross-correlation $\hat{A}_{\mathrm{r}}\left[k_{1}\right]=\mathbf{s}_{k_{1}}^{H} \mathbf{y}_{k_{1}}^{1} /\left\|\mathbf{s}_{k_{1}}\right\|^{2}$. The waveform used for cancellation is

$$
\hat{r}_{k_{1}}(\mathrm{t})=\sum_{m=0}^{n-1} \hat{\mathbf{r}}_{k_{1}}^{\mathrm{ok}}[m] c_{k_{1}, m}\left(\mathrm{t}-m T-\tau_{k_{1}}\right) .
$$

\section{Receiver's Operation: The Second Iteration}

As a consequence of the non-error free performance of the adopted coding scheme, some users may be unsuccessfully decoded during the first SIC iteration. Intuitively, this may occur more frequently for less powerful encoders, usually associated with the transmission of very short packets. For long-packet communications, the number of users unsuccessfully decoded at the end of the first iteration will be practically zero if a proper energy allocation is designed. Nonetheless, since in this manuscript we do not consider a particular encoder except for numerical analysis, we continue with a general model in which the number of users to be processed is $K_{2}<K$.

We proceed in the same way as in the first SIC iteration. Firstly, we define $1 \leq k_{2} \leq K_{2}$ to index the users undergoing a second decoding attempt, and secondly, we relate their indices with those in the first SIC iteration using

$$
\phi: k_{1}^{\prime} \longleftrightarrow k_{2} .
$$

$k_{1}^{\prime} \subset\{1, \ldots, K\}$ is the index set only for users that survive after the first decoding attempt. Then: $\phi^{-1}\left[k_{2}\right]$ produces the index of user $k_{2}$ in the first SIC iteration; and vice-versa, $\phi\left[k_{1}^{\prime}\right]$ gets the new index of user $k_{1}^{\prime}$.

The output of the symbol despreader for user $k_{2}$, which was indexed as $k=\phi^{-1}\left[k_{2}\right]$ in the first SIC iteration, reads

$$
\left.\mathbf{y}_{k}^{2}=A_{\mathrm{r}}[k] \mathbf{s}_{k}+\sqrt{N_{t}^{2}\left[k_{2}\right.}\right] \mathbf{w}_{k}^{2} \quad \text { with } \quad k=\phi^{-1}\left[k_{2}\right] .
$$

Now, $\mathbf{w}_{k}^{2}$ is a unit-variance Gaussian term comprising the aggregate interference from all the imperfectly canceled users in the first SIC iteration plus that from processed and yet unprocessed users in the second SIC iteration, and thermal noise. $N_{t}^{2}\left[k_{2}\right]$ is the noise plus interference level at stage $k_{2}$ in the second iteration. After channel decoding $\hat{\mathbf{b}}_{k}=f^{-1}\left(\mathbf{y}_{k}^{2}\right)$, packet error is determined by checking the CRC. If so, the received signal from user $k_{2}\left(k=\phi^{-1}\left[k_{2}\right]\right.$ according to the indexing in the first iteration) is reconstructed as $\hat{\mathbf{r}}_{k}^{\mathrm{ok}}=\hat{A}_{\mathrm{r}}[k] \mathbf{s}_{k}$ with $\hat{A}_{\mathrm{r}}[k]=\mathbf{s}_{k}^{H} \mathbf{y}_{k}^{2} /\left\|\mathbf{s}_{k}\right\|^{2}$, and its resulting waveform $\hat{r}_{k}(\mathrm{t})$ is canceled from $y(\mathrm{t})$ in (2).

We now analyze the similarities and dissimilarities between the first and the second SIC iterations for a user $k_{1}^{\prime}$ that undergoes a second decoding attempt at stage $k_{2}^{\prime}=\phi\left[k_{1}^{\prime}\right]$. In this case, the only differential term is $\mathbf{w}_{k_{1}^{\prime}}^{1}$ in (4) and $\mathbf{w}_{k_{1}^{\prime}}^{2}$ in (7) and their corresponding variances. Their dissimilarities are due to the amount of interference canceled between the processing of user $k_{1}^{\prime}$ in the first iteration (stage $k_{1}^{\prime}$ ) and in the second iteration (stage $k_{2}^{\prime}$ ). Qualitatively, the second attempt will succeed more frequently when $N_{t}^{2}\left[k_{2}^{\prime}\right] \ll N_{t}^{1}\left[k_{1}^{\prime}\right]$ and $\mathbf{w}_{k_{1}^{\prime}}^{1}, \mathbf{w}_{k_{1}^{\prime}}^{2}$ substantially differ. Quantitatively, the decoder's performance versus $N_{t}^{1}\left[k_{1}^{\prime}\right]-N_{t}^{2}\left[k_{2}^{\prime}\right]$ is analyzed below.

\section{SINR-BASEd SYSTEM MODEL}

We derive expressions for the previous system in terms of the SINR of each user after symbol despreading. The expressions we adopt contain accurate models of the most relevant features of successive decoding [28], [29]: non-ideal channel decoding and imperfect SIC cancellation. The following sections describe concrete analyses for each iteration.

\section{A. The First SIC Iteration}

Firstly, we analyze the output of the $k_{1}$-th user despreader $\mathbf{y}_{k_{1}}^{1}$ given by equation (4). When long spreading codes are utilized, the cross-correlation between the spreading waveform of user $k_{1}, c_{k_{1}, m}(t)$, and other waveforms $c_{k \neq k_{1}, m}(t)$ entail the reasonable assumption of considering multiple access interference as uncorrelated Gaussian noise. Thus, for system analysis, interference can be added to thermal noise under the decorrelation gain $\frac{1}{N}$. The SINR for the $k_{1}$-th user is

$$
\Gamma^{1}\left[k_{1}\right]=\frac{E_{\mathrm{r}}\left[k_{1}\right]}{N_{t}^{1}\left[k_{1}\right]}
$$

the ratio between the received symbol energy from user $k_{1}, E_{\mathrm{r}}\left[k_{1}\right] \triangleq\left|A_{\mathrm{r}}\left[k_{1}\right]\right|^{2}=E_{\mathrm{x}}\left[k_{1}\right] h\left[k_{1}\right]$, and the noise plus interference level at stage $k_{1}$.

Secondly, we adopt a reasonable model for the $k_{1}$-th user decoding and packet error detection in terms of the univariate PER versus SINR curve PER $\left[\Gamma^{1}\right]$ evaluated at $\Gamma^{1}\left[k_{1}\right]$. Accordingly, after checking the CRC, user $k_{1}$ is unsuccessfully 
decoded with probability $\operatorname{PER}\left[\Gamma^{1}\left[k_{1}\right]\right]$ or successfully decoded with complementary probability. The computation of $\operatorname{PER}\left[\Gamma^{1}\right]$ at the SINR $\Gamma^{1} \triangleq E_{\mathrm{r}} / N_{t}^{1}$ is undertaken, separately, by averaging the number of erroneous decoding trials of an encoded vector $\mathbf{b}$ received with symbol energy $E_{\mathrm{r}}$, random phase $\nu$, and contaminated by Gaussian noise $\mathbf{w}^{1} \sim \mathcal{C N}\left(\mathbf{0}_{n}, \mathbf{I}_{n}\right)$, as

$$
\begin{aligned}
\mathbf{y}^{1} & =\sqrt{E_{\mathrm{r}}} e^{j \nu} f(\mathbf{b})+\sqrt{N_{t}^{1}} \mathbf{w}^{1}, \\
\operatorname{PER}\left[\Gamma^{1}\right] & \triangleq \operatorname{Pr}\left[f^{-1}\left(\mathbf{y}^{1}\right) \neq \mathbf{b}\right] .
\end{aligned}
$$

Thirdly, imperfect cancellation at the waveform level is assumed, since the reconstructed waveform for user $k_{1}, \hat{r}_{k_{1}}(\mathrm{t})$, may not coincide with its received waveform. Our model considers that, after every user cancellation, a fraction $\varepsilon\left[\Gamma^{1}\right]$ of its energy remains uncanceled. The computation of $\varepsilon\left[\Gamma^{1}\right]$ is carried out separately, assuming that, after a successful $\mathrm{CRC}$, the magnitude of imperfect cancellation is mainly given by the accuracy of complex amplitude estimation of the symbols. More specifically, we compute it by reconstructing the received sequence from a successfully decoded user as $\hat{\mathbf{r}}^{\mathrm{ok}}$ and by evaluating

$$
\varepsilon\left[\Gamma^{1}\right] \triangleq \frac{\mathbb{E}\left[\left\|\hat{\mathbf{r}}^{\mathrm{ok}}-\sqrt{E_{\mathrm{r}}} e^{j \nu} f(\mathbf{b})\right\|^{2}\right]}{\mathbb{E}\left[\left\|\sqrt{E_{\mathrm{r}}} f(\mathbf{b})\right\|^{2}\right]} .
$$

With the characterization explained above, the $k_{1}$-th user SINR after symbol despreading is

$$
\Gamma^{1}\left[k_{1}\right]=\frac{E_{\mathrm{r}}\left[k_{1}\right]}{N_{0}+\frac{\theta}{N} \sum_{k<k_{1}} \epsilon_{1}[k] E_{\mathrm{r}}[k]+\frac{\theta}{N} \sum_{k>k_{1}} E_{\mathrm{r}}[k]} .
$$

$N_{0}$ is the power spectral density of thermal noise, and the two remaining terms correspond to the interference from yet processed users (second term) and from the still unprocessed ones (third term). The factor $\theta$ models relevant time-variations of $\tau[1], \ldots, \tau[K]$ due to asynchronous user operations; $\frac{\theta}{N}$ is the second moment of the cross-correlation between signature waveforms of different users [28], [34]. Finally, according to the SIC policy described in Section II-B, we model channel decoding and the binary CRC decisions by introducing one binary random variable $\epsilon_{1}\left[k_{1}\right]$ for each user along with the assumption of independent decoding between different users: $\epsilon_{1}\left[k_{1}\right]$ equals 1 when the CRC does not check out (with probability $\left.\operatorname{PER}\left[\Gamma^{1}\left[k_{1}\right]\right]\right)$, or $\varepsilon\left[\Gamma^{1}\left[k_{1}\right]\right]$ when user $k_{1}$ is successfully decoded (correct CRC).

\section{B. The Second SIC Iteration}

Recall that users with the received symbol energies $E_{\mathrm{r}}^{2}[1 \leq$ $k_{2} \leq K_{2}$ ] undergo a second decoding attempt. We use the indexing relationship (6) to obtain their symbol energies from the initial symbol energy distribution as

$$
E_{\mathrm{r}}^{2}\left[k_{2}\right]=E_{\mathrm{r}}\left[\phi^{-1}\left[k_{2}\right]\right] .
$$

Next, we continue with the Gaussian interference assumption for the computation of the $k_{2}$-th user SINR, so that

$$
\Gamma^{2}\left[k_{2}\right]=\frac{E_{\mathrm{r}}^{2}\left[k_{2}\right]}{N_{t}^{2}\left[k_{2}\right]}=\frac{E_{\mathrm{r}}\left[\phi^{-1}\left[k_{2}\right]\right]}{N_{t}^{2}\left[k_{2}\right]} .
$$

To model channel decoding in the second SIC iteration, we adopt the accurate proposal in [29] where the success/error behaviors are evaluated in terms of the SINRs of each user at every iteration. More specifically, we add an additional argument to the univariate PER PER $\left[\Gamma^{1}\right]$ to account for persistent decoding operations for the same user, as the $b i$ variate function $\mathrm{PER}_{2}\left[\Gamma^{1}, \Gamma^{2}\right]$. The latter is understood as the packet error probability for a user with the SINR $\Gamma^{2}$ unsuccessfully decoded in the first iteration under the SINR $\Gamma^{1}$. Its computation substantially differs from that explained in Section III-A for the univariate PER. In this case, we consider that the term $\mathbf{w}^{1}$ in (9a) is decomposed into two independent Gaussian noise terms under the linear model

$$
\sqrt{N_{t}^{1}} \mathbf{w}^{1} \triangleq \sqrt{N_{t}^{2}} \mathbf{w}^{2}+\sqrt{N_{t}^{1}-N_{t}^{2}} \mathbf{w}^{c},
$$

with $\mathbf{w}^{2}, \mathbf{w}^{c} \sim \mathcal{C N}\left(\mathbf{0}_{n}, \mathbf{I}_{n}\right)$, such that the resulting signals after symbol despreading in each SIC iteration can be expressed, respectively, as

$$
\begin{aligned}
& \mathbf{y}^{1}=\sqrt{E_{\mathrm{r}}} e^{j \nu} f(\mathbf{b})+\sqrt{N_{t}^{2}} \mathbf{w}^{2}+\sqrt{N_{t}^{1}-N_{t}^{2}} \mathbf{w}^{c}, \\
& \mathbf{y}^{2}=\sqrt{E_{\mathrm{r}}} e^{j \nu} f(\mathbf{b})+\sqrt{N_{t}^{2}} \mathbf{w}^{2},
\end{aligned}
$$

under the SINRs $\Gamma^{1}=E_{\mathrm{r}} / N_{t}^{1}$ and $\Gamma^{2}=E_{\mathrm{r}} / N_{t}^{2} ; \Gamma^{2} \geq \Gamma^{1}$. Note that $\mathbf{y}^{1}$ corresponds exactly to (9a), while for the computation of $\mathbf{y}^{2}$, the noise term $\mathbf{w}^{c}$ has been subtracted to model an interference cancellation operation of energy $N_{t}^{1}-N_{t}^{2}$. Hence, the bivariate PER is computed as

$$
\mathrm{PER}_{2}\left[\Gamma^{1}, \Gamma^{2}\right] \triangleq \operatorname{Pr}\left[f^{-1}\left(\mathbf{y}^{2}\right) \neq \mathbf{b} \mid f^{-1}\left(\mathbf{y}^{1}\right) \neq \mathbf{b}\right] .
$$

The SINR after symbol despreading for the $k_{2}$-th user in the second SIC iteration is

$$
\Gamma^{2}\left[k_{2}\right]=\frac{E_{\mathrm{r}}^{2}\left[k_{2}\right]}{N_{0}+\xi_{1}+\frac{\theta}{N} \sum_{k<k_{2}} \epsilon_{2}[k] E_{\mathrm{r}}^{2}[k]+\frac{\theta}{N} \sum_{k>k_{2}} E_{\mathrm{r}}^{2}[k]}
$$

where $\xi_{1}$ contains the interference generated by successfully decoded and imperfectly canceled users after the first SIC iteration; the third and fourth terms correspond to the contributions of users processed during the current iteration. In this case, for a given user $k_{2}$, we define the vector $\Gamma^{2}\left[k_{2}\right]$ of the SINRs experienced by user $k_{2}$ in each SIC iteration as $\Gamma^{2}\left[k_{2}\right] \triangleq\left[\Gamma^{1}\left[\phi^{-1}\left[k_{2}\right]\right], \Gamma^{2}\left[k_{2}\right]\right]$, and that we use to evaluate the decoding performance of user $k_{2}$ in the second iteration as argument of the bivariate PER. In (17), $\epsilon_{2}[k]$ is the random variable modeling the second decoding attempt for user $k$, which fails $\left(\epsilon_{2}[k]=1\right)$ with probability $\operatorname{PER}_{2}\left[\Gamma^{2}[k]\right]$ and succeeds $\left(\epsilon_{2}[k]=\varepsilon\left[\Gamma^{2}[k]\right]\right)$ with complementary probability when the CRC checks out. Under successful decoding, we consider the fraction $\varepsilon\left[\Gamma^{2}[k]\right]$ of the $k$-th user's energy remaining uncanceled.

\section{Accurate Approximation to the Bivariate PER}

The introduction of such a bivariate PER, although modeling the exact behavior of the two-iteration SIC, poses some difficulties due to complexity issues in its computation. The 
authors in [29] addressed this issue, by suggesting the following bound to approximate the bivariate function:

$$
\operatorname{PER}_{2}\left[\Gamma^{1}, \Gamma^{2}\right] \leq \frac{\operatorname{PER}\left[\Gamma^{2}\right]}{\operatorname{PER}\left[\Gamma^{1}\right]} .
$$

The term on the right, although it does not characterize the exact performance of the second decoding attempt, provides a very competitive solution at much less computational cost that lets us adopt such a bound with strict equality. More specifically, it was shown in [29] that the bound is very tight for SINR gains higher than only half a dB. This way, the computation of the bivariate PER is then facilitated by adopting an analytic form or a lookup table for the univariate PER curve associated with the adopted coding scheme. This bound, in addition to computational benefits, provides insight into the performance attained in the second decoding attempt, as for the following two examples: (i) the decoding performance of the first SIC iteration does not improve when no interference is canceled between iterations, since $\Gamma^{2}=\Gamma^{1}$ and $\operatorname{PER}_{2}\left[\Gamma^{1}, \Gamma^{2}\right]=1$; and (ii) the performance of the new decoding trial strongly depends on PER $\left[\Gamma^{2}\right]$ when a high amount of interference is canceled between iterations, and so $\Gamma^{2} \gg \Gamma^{1}$, and its true performance is not worse than the right term of (18).

\section{System Analysis in the Infinite User Regime}

The objective pursued in this section is to analyze the userlimit behavior of the two-iteration SIC firstly, by approximating the system behavior for a massively deployed network, and secondly, by turning the randomness caused by non-ideal decoding to deterministic expressions in favor of our first goal. Then, we show in the sequel the deterministic forms of the previous system model in the regime of large number of users $K$ and large spreading gain $N$, at the linear scaling $\alpha \triangleq K / N$.

\section{A. Infinite User Analysis for the First SIC Iteration}

Recall the analyses in previous works [26], [27] in which, instead of using the standard indexing $k_{1}=1,2, \ldots, K$, the normalized indexing (with respect to the number of users)

$$
t_{1} \triangleq \lim _{K \rightarrow \infty} k_{1} / K
$$

is adopted to turn the ordered distributions to functions of the continuous user-variable $t_{1}$. For the function capturing the user-channel gains, we have, correspondingly

$$
h\left(t_{1}\right) \triangleq \lim _{K \rightarrow \infty} h\left[t_{1} K\right],
$$

which, if it varies smoothly, constitutes a continuous function (profile) in the infinite user regime. Moreover, the rest of userdistributions are turned into continuous functions of the uservariable $0 \leq t_{1} \leq 1$. The SINR profile in the first SIC iteration is (21), where the noise plus interference profile $N_{t}^{1}\left(t_{1}\right)$ can be obtained by introducing the (traffic load) variable $\alpha$ and by substituting the summations in the denominator of (11) by integrals under $K^{-1} \rightarrow \mathrm{d} \tau$, as resulted in (22):

$$
\begin{aligned}
\Gamma^{1}\left(t_{1}\right) & =\frac{E_{\mathrm{r}}\left(t_{1}\right)}{N_{t}^{1}\left(t_{1}\right)} \\
N_{t}^{1}\left(t_{1}\right) & =N_{0}+\alpha \theta \int_{0}^{t_{1}} \epsilon_{1}(\tau) E_{\mathrm{r}}(\tau) \mathrm{d} \tau+\alpha \theta \int_{t_{1}}^{1} E_{\mathrm{r}}(\tau) \mathrm{d} \tau .
\end{aligned}
$$

In the previous equation, since in the interval $\left[0, t_{1}\right]$ the contribution of infinite users are averaged, we invoke the law of large numbers and satisfy Kolmogorov's condition to ensure that each of the random variables $\epsilon_{1}$ can be substituted by its expectation $1-\left(1-\varepsilon\left[\Gamma^{1}\right]\right)\left(1-\operatorname{PER}\left[\Gamma^{1}\right]\right)$. This yields, after some manipulations [29], in a simplified expression for the noise plus interference profile in terms of the exponential evolution (23a), with $\Phi\left[\Gamma^{1}\right] \triangleq \theta\left(1-\varepsilon\left[\Gamma^{1}\right]\right) \Gamma^{1}\left(1-\operatorname{PER}\left[\Gamma^{1}\right]\right)$ and the initial noise plus interference term (23b):

$$
\begin{aligned}
& N_{t}^{1}\left(t_{1}\right)=N_{t}^{1}(0) \exp \left(-\alpha \int_{0}^{t_{1}} \Phi\left[\Gamma^{1}(\tau)\right] \mathrm{d} \tau\right), \\
& N_{t}^{1}(0)=N_{0}+\alpha \theta \int_{0}^{1} E_{\mathrm{x}}(\tau) h(\tau) \mathrm{d} \tau .
\end{aligned}
$$

\section{B. Infinite User Analysis for the Second SIC Iteration}

At the beginning of the second SIC iteration, the remaining traffic load asymptotically yields

$$
\alpha_{2}=\frac{K_{2}}{N} \rightarrow \alpha \overline{\operatorname{per}}_{1} ; \overline{\operatorname{per}}_{1} \triangleq \int_{0}^{1} \operatorname{PER}\left[\Gamma^{1}(\tau)\right] \mathrm{d} \tau .
$$

We assume that $\alpha_{2}$ remains constant as $K_{2} \rightarrow \infty$. With the aid of [29], this assumption is reasonable for the analysis of the first iteration when $K$ is a finite large number, but may be less precise for the second iteration as it operates with fewer users. For that reason, and overcoming this weakness, we continue with an asymptotically constant traffic load $\alpha_{2}$ and, in contrast to [27], we address the validation of such an assumption in Section VI via simulations.

Identically as in the first iteration, we define the following user-index with which to operate in the second iteration

$$
t_{2} \triangleq \lim _{K_{2} \rightarrow \infty} k_{2} / K_{2}
$$

with the advantage that, in the infinite user case, $K_{2}$ is no longer random, and thus, $0 \leq t_{2} \leq 1$ is a deterministic userindex used to operate with continuous functions, as with the relationship (12) now turned into

$$
E_{\mathrm{r}}^{2}\left(t_{2}\right)=E_{\mathrm{r}}\left(\phi^{-1}\left(t_{2}\right)\right) .
$$

The user-indices $t_{1}$ and $t_{2}$ can be mutually related recalling that, after the first iteration, each interval of users $\mathrm{d} t_{1}$ can be split into the fraction $\operatorname{PER}\left[\Gamma^{1}\left(t_{1}\right)\right]$ of users unsuccessfully decoded plus the fraction $1-\operatorname{PER}\left[\Gamma^{1}\left(t_{1}\right)\right]$ that are successfully decoded: $\mathrm{d} t_{1}=\operatorname{PER}\left[\Gamma^{1}\left(t_{1}\right)\right] \mathrm{d} t_{1}+\left(1-\operatorname{PER}\left[\Gamma^{1}\left(t_{1}\right)\right]\right) \mathrm{d} t_{1}$. Then, since the second SIC iteration operates only with the users unsuccessfully decoded after the first iteration, the new user-index $t_{2}$ is given only by the first term. We define

$$
\mathrm{d} t_{2} \triangleq \frac{1}{\overline{\operatorname{per}}_{1}} \cdot \operatorname{PER}\left[\Gamma^{1}\left(t_{1}\right)\right] \mathrm{d} t_{1},
$$

which, after solving the previous differential equation with the initial condition $t_{2}=0$ when $t_{1}=0$, gives us the long awaited relationship between $t_{1}$ and $t_{2}: t_{2}=\phi\left(t_{1}\right)$ with

$$
\phi(t) \triangleq \frac{1}{\overline{\overline{\operatorname{per}}_{1}}} \int_{0}^{t} \operatorname{PER}\left[\Gamma^{1}(\tau)\right] \mathrm{d} \tau .
$$


With all of this, the SINR profile after symbol despreading in the second SIC iteration given by (13) is turned into any of the following equivalent forms:

$$
\Gamma^{2}\left(t_{2}\right)=\frac{E_{\mathrm{r}}^{2}\left(t_{2}\right)}{N_{t}^{2}\left(t_{2}\right)} \quad, \quad \Gamma^{2}\left(\phi\left(t_{1}\right)\right)=\frac{E_{\mathrm{r}}\left(t_{1}\right)}{N_{t}^{2}\left(\phi\left(t_{1}\right)\right)} .
$$

For the substantial benefit of our subsequent rationales, we adopt the second form $\Gamma^{2}\left(\phi\left(t_{1}\right)\right)$ for the SINR profile. Its denominator is the noise plus interference profile, whose evolution can be characterized, following identical rationales as in [29], by the following exponential expression with $\Psi\left[\Gamma^{1}, \Gamma^{2}\right] \triangleq \theta\left(1-\varepsilon\left[\Gamma^{2}\right]\right) \Gamma^{2}\left(\operatorname{PER}\left[\Gamma^{1}\right]-\operatorname{PER}\left[\Gamma^{2}\right]\right)$ and where the initial noise plus interference term $N_{t}^{2}(\phi(0))=N_{t}^{2}(0)$ coincides, in the asymptotic large-user regime, with the last noise plus interference term in the first iteration, as:

$$
\begin{aligned}
N_{t}^{2}\left(\phi\left(t_{1}\right)\right) & =N_{t}^{2}(0) \exp \left(-\alpha \int_{0}^{t_{1}} \Psi\left[\Gamma^{2}(\phi(\tau))\right] \mathrm{d} \tau\right), \\
N_{t}^{2}(0) & =N_{t}^{1}(0) \exp \left(-\alpha \int_{0}^{1} \Phi\left[\Gamma^{1}(\tau)\right] \mathrm{d} \tau\right) .
\end{aligned}
$$

The SINR vector is $\Gamma^{2}\left(\phi\left(t_{1}\right)\right)=\left[\Gamma^{1}\left(t_{1}\right), \Gamma^{2}\left(\phi\left(t_{1}\right)\right)\right]$.

\section{Reliability Figures of Merit}

We study two figures of merit in terms of communication error performance that follow a user-centric or a networkcentric criterion. We analyze them for the one-iteration and the two-iteration SIC receivers separately.

For the one-iteration SIC, the individual packet error probability coincides with the PER of each user (31), and the network packet error probability (network PER) results from averaging the individual error probabilities as in (32):

$$
\begin{aligned}
\operatorname{per}\left(t_{1}\right) & =\operatorname{PER}\left[\Gamma^{1}\left(t_{1}\right)\right] \quad \text { in } \quad 0 \leq t_{1} \leq 1, \\
\overline{\operatorname{per}}_{1} & =\int_{0}^{1} \operatorname{per}(\tau) \mathrm{d} \tau=\int_{0}^{1} \operatorname{PER}\left[\Gamma^{1}(\tau)\right] \mathrm{d} \tau .
\end{aligned}
$$

For the two-iteration SIC, the network packet error probability coincides with the product of the average packet error probabilities over all users in every iteration. Then, we have

$$
\overline{\operatorname{per}}_{2}=\overline{\operatorname{per}}_{1} \int_{0}^{1} \operatorname{PER}_{2}\left[\boldsymbol{\Gamma}^{2}(\tau)\right] \mathrm{d} \tau,
$$

which can be simplified by substituting $\mathrm{PER}_{2}\left[\Gamma^{1}, \Gamma^{2}\right]=$ $\operatorname{PER}\left[\Gamma^{2}\right] / \operatorname{PER}\left[\Gamma^{1}\right]$, and by applying the change of variable $\tau=\phi(t)$ to the previous integral. This gives

$$
\overline{\operatorname{per}}_{2}=\int_{0}^{1} \operatorname{PER}\left[\Gamma^{2}(\phi(t))\right] \mathrm{d} t,
$$

and thus, the individual PER experienced by each user after two SIC iterations is given by its integrand, and reads

$$
\operatorname{per}\left(t_{1}\right) \triangleq \operatorname{PER}\left[\Gamma^{2}\left(\phi\left(t_{1}\right)\right)\right] \quad \text { in } \quad 0 \leq t_{1} \leq 1
$$

\section{ASYMPTOTIC OPTIMIZATION}

This section addresses the energy allocation design, which, from the energy efficiency point of view, deals with the minimization of the average energy transmitted by all users, denoted herein energy expenditure,

$$
\bar{E} \triangleq \int_{0}^{1} E_{\mathrm{x}}(t) \mathrm{d} t
$$

Since iterative SIC substantially outperforms one-iteration SIC when, instead of working in the infinite blocklength regime, communication is performed under short packets, we continue our analysis following an order that precisely highlights this performance improvement; in our case, we elaborate on the reduction of energy expenditure. More specifically, we analyze the infinite and the finite blocklength regimes for coding schemes of the same rate $R$. Moreover, we incorporate the load regulation index $t_{*} \leq 1$ into our system analysis, which allows for setting the satellite amplifier's operating point below saturation or controlling energy expenditure in high activity load situations, by deactivating the transmission of users with poor channel gains, as follows

$$
E_{\mathrm{x}}\left(t_{1}\right)=0 \text { in } t_{1}>t_{*} .
$$

We also study, as a relevant part of this work, the minimization of energy expenditure subject to either fair or unfair reliability for active transmitters:

1) Unfair allocation: Minimization of energy expenditure subject to the network-centric (average) constraint $c<1$

$$
\frac{1}{t_{*}} \int_{0}^{t_{*}} \operatorname{per}(t) \mathrm{d} t \leq c
$$

2) Fair allocation: Minimization of energy expenditure subject to the user-centric (individual) constraint

$$
\operatorname{per}\left(t_{1}\right) \leq c \quad \text { in } \quad 0 \leq t_{1} \leq t_{*} .
$$

\section{A. Communication in the Infinite Blocklength Regime}

We first analyze the case in which the blocklength $n_{e}$ goes to infinity and the SIC's uncanceled energy factor is $\varepsilon\left[\Gamma^{1}\right]=\varepsilon$. In this case, the adopted capacity-achieving coding scheme shows a PER characteristic expressed in terms of the unitary step function $u(x)$ as

$$
\operatorname{PER}\left[\Gamma^{1}\right]=1-u\left(\Gamma^{1}-\Gamma_{\text {th }}\right),
$$

where transmission is reliable for free only if $\Gamma^{1} \geq \Gamma_{\text {th }}$ with $\Gamma_{\text {th }} \triangleq 2^{R}-1$ if $R$ is given in bits per channel use. Since error free decoding is possible if energy allocation is properly designed, one-iteration SIC suffices to decode all active users $0 \leq t \leq t_{*}$. In this case, both reliability constraints are attained at the same time as long as users are allocated as

$$
\Gamma^{1}\left(t_{1}\right) \geq \Gamma_{\mathrm{th}}
$$

and, in particular, to minimize energy expenditure, the active users $0 \leq t_{1} \leq t_{*}$ shall be allocated at the SINR $\Gamma^{1}\left(t_{1}\right)=\Gamma_{\text {th }}$. The decoding-cancellation characteristic for this SIC receiver is $\Phi_{p}\left[\Gamma^{1}\right]=\theta(1-\varepsilon) \Gamma^{1}$. The received symbol energy profile is $E_{\mathrm{r}}\left(t_{1}\right)=\Gamma_{\mathrm{th}} N_{t}\left(t_{1}\right)=\Gamma_{\mathrm{th}} N_{t}^{1}(0) \exp \left(-\alpha \Phi_{p}\left[\Gamma_{\mathrm{th}}\right] t_{1}\right)$ in $0 \leq$ 
$t_{1} \leq t_{*}$, with the initial noise plus interference term obtained by computing $N_{t}^{1}(0)$ from (23b) and by solving the integral of an exponential function:

$$
N_{t}^{1}(0)=\frac{N_{0}(1-\varepsilon)}{\exp \left(-\alpha \Phi_{p}\left[\Gamma_{\mathrm{th}}\right] t_{*}\right)-\varepsilon} .
$$

Since $N_{t}^{1}(0)>0$, the following achievable region is defined

$$
\alpha t_{*} \leq \frac{1}{\Phi_{p}\left[\Gamma_{\mathrm{th}}\right]} \log \left(\frac{1}{\varepsilon}\right) .
$$

Therefore, for an achievable $\alpha t_{*}$, the transmitted symbol energy for any active user $0 \leq t_{1} \leq t_{*}$ is computed as

$$
E_{\mathrm{x}}\left(t_{1}\right)=\frac{\Gamma_{\mathrm{th}} N_{t}^{1}(0)}{h\left(t_{1}\right)} \exp \left(-\alpha \Phi_{p}\left[\Gamma_{\mathrm{th}}\right] t_{1}\right)
$$

\section{B. Communication with Short Packets: Unfair Energy Alloca- tion and One-Iteration SIC}

We now consider a short-packet communication setting handled by a one-iteration SIC whose decoding strategy is subject to a non-error free performance. Then, we minimize, under $E_{\mathrm{x}}(t)$, the average energy over all users while guaranteeing the average reliability over active users:

$$
\begin{gathered}
\min _{E_{\mathrm{x}}(t)} \int_{0}^{t_{*}} E_{\mathrm{x}}(t) \mathrm{d} t \quad \text { s.t. } \int_{0}^{t_{*}} \operatorname{PER}\left[\frac{E_{\mathrm{x}}(t) h(t)}{N_{t}^{1}(t)}\right] \mathrm{d} t=t_{*} c \\
\text { s.t. eqs. (23a) }-(23 \mathrm{~b})
\end{gathered}
$$

We solve this problem is by turning integrals into Riemannian sums under (large) $r$ intervals $t_{1}^{1}, t_{1}^{2}, \ldots, t_{1}^{r}$ and, since the involved functions are twice continuously differentiable, by resorting to sequential quadratic programming (SQP) with the initialization $\Gamma^{1}\left(t_{1}^{i}\right)=\mathrm{PER}^{-1}[c]$ for $1 \leq i \leq r$. To apply SQP, we need the gradient and the Hessian of the profit and constraints. We leverage the calculus of variations and substitute gradients by variational derivatives sampled at each $t_{1}^{i}$ and scaled by $\frac{1}{r}$. The variational derivatives are shown in Appendix I; the Hessian is computed numerically.

\section{Communication with Short Packets: Fair Energy Allocation and One-Iteration SIC}

When the user-centric reliability constraint is superimposed and the receiver operates under one-iteration SIC, we have

$$
\operatorname{per}\left(t_{1}\right)=\operatorname{PER}\left[\Gamma^{1}\left(t_{1}\right)\right]=c \quad \text { in } \quad 0 \leq t_{1} \leq t_{*} .
$$

All users are allocated to equal SINRs $\Gamma_{c} \triangleq \operatorname{PER}^{-1}[c]$. The SINR profile is the constant function $\Gamma^{1}\left(t_{1}\right)=\Gamma_{c}$ in $0 \leq$ $t_{1} \leq t_{*}$, in which case the same derivation as in Section V-A can be applied to obtain the transmitted symbol energies but substituting $\Gamma_{\text {th }}$ by $\Gamma_{c}$ and $\varepsilon$ by $\varepsilon\left[\Gamma_{c}\right]$.
D. Communication with Short Packets: Unfair Energy Allocation and Two-Iteration SIC

We introduce, for the first time in this manuscript, the optimization problem under the two-iteration SIC receiver:

$$
\begin{aligned}
\min _{E_{\mathrm{x}}(t)} \int_{0}^{t_{*}} E_{\mathrm{x}}(t) \mathrm{d} t & \text { s.t. } \int_{0}^{t_{*}} \operatorname{PER}\left[\frac{E_{\mathrm{x}}(t) h(t)}{N_{t}^{2}(\phi(t))}\right] \mathrm{d} t=t_{*} c \\
\text { s.t. eqs. (23a) }-(23 \mathrm{~b}) & \text { s.t. eqs. (30a)-(30b) }
\end{aligned}
$$

We solve the problem under SQP, and computing gradients analytically from the variational derivatives of the cost function and the constraints. Appendix II presents analytic forms for the computed variational derivatives.

\section{E. Communication with Short Packets: Fair Energy Allocation under Two-Iteration SIC}

When the user-centric reliability constraint is superimposed and the receiver operates under a two-iteration SIC, we have

$$
\operatorname{per}\left(t_{1}\right)=\operatorname{PER}\left[\Gamma^{2}\left(\phi\left(t_{1}\right)\right)\right]=c \quad \text { in } \quad 0 \leq t_{1} \leq t_{*} .
$$

Then, the optimum SINR profile in the second SIC iteration is $\Gamma^{2}\left(\phi\left(t_{1}\right)\right)=\Gamma_{\mathrm{c}}$ in $0 \leq t_{1} \leq t_{*}\left(\right.$ with $\left.\Gamma_{\mathrm{c}}=\mathrm{PER}^{-1}[c]\right)$. Now, we find the symbol energy profile that generates such a uniform SINR profile in the second SIC iteration: we sample $t_{1}$ in the $r$ intervals $t_{1}^{1}, t_{1}^{2}, \ldots, t_{1}^{r}$; we set $N_{t}^{1}\left(t_{1}^{1}\right) \rightarrow N_{t}^{1}(0)$ and $N_{t}^{2}\left(\phi\left(t_{1}^{1}\right)\right) \rightarrow N_{t}^{2}(\phi(0))$; we assume temporarily knowledge of $N_{t}^{1}(0)$ and $N_{t}^{2}(\phi(0))$, and find their true values by bisection searches. The received symbol energies are computed as

$$
E_{\mathrm{r}}\left(t_{1}^{i}\right)=\Gamma_{\mathrm{c}}^{2} \cdot N_{t}^{2}\left(\phi\left(t_{1}^{i}\right)\right) \quad \text { in } \quad 1 \leq i \leq r,
$$

wherein each element of the noise plus interference term is computed recursively as

$$
\begin{aligned}
N_{t}^{1}\left(t_{1}^{i+1}\right) & =N_{t}^{1}\left(t_{1}^{i}\right) \exp \left(-\frac{\alpha}{r} \Phi\left[\Gamma^{1}\left(t_{1}^{i}\right)\right]\right), \\
N_{t}^{2}\left(\phi\left(t_{1}^{i+1}\right)\right) & =N_{t}^{2}\left(\phi\left(t_{1}^{i}\right)\right) \exp \left(-\frac{\alpha}{r} \Psi\left[\Gamma^{1}\left(\phi\left(t_{1}^{i}\right)\right), \Gamma_{\mathrm{c}}\right]\right) .
\end{aligned}
$$

\section{Simulations}

The simulations comprise, in Section VI-A, a theoretical study of a semi-realistic satellite return channel in which the geographic distribution users is approximated through channel power gains log-normally distributed, and secondly, in Section VI-B, the accuracy assessment of the analyzed setting for a low-level implementation of the adopted SIC receiver.

\section{A. Theoretical Study}

Our theoretical study evaluates the energy expenditure (36) when the network handles asymptotically many users. For system encoding and modulation, we evaluate theoretical coding schemes with achievable second order coding rates [22] of $R=2 / 3$ bits/symbol and many blocklengths $n_{e}$. As a practical coding scheme, we consider the turbo code from Digital Video Broadcasting (DVB-RCS) [35] with 128 information bits, coding rate $1 / 3$, and QPSK modulation (payload of $n_{e}=192$ symbols plus $n_{o}=28$ preamble symbols); decoding is performed under 20 max-log-map iterations. Their univariate 


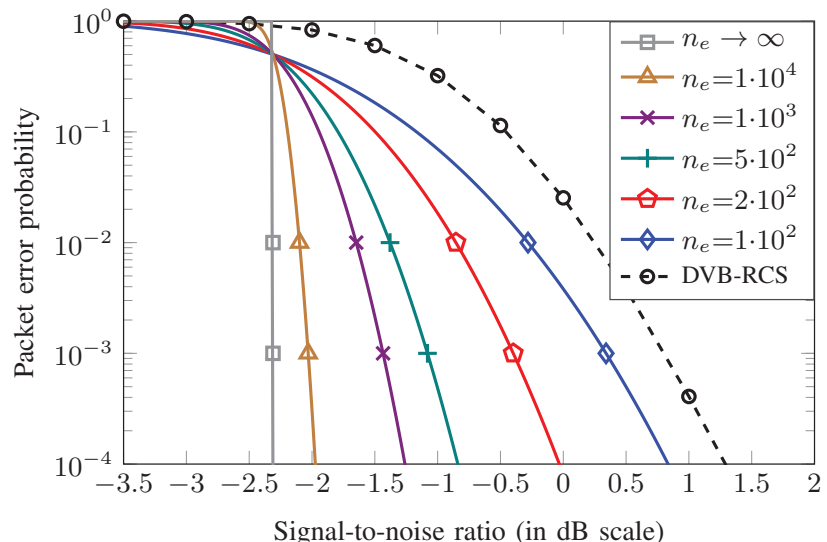

Fig. 1. Univariate PER curve: $\operatorname{PER}[\Gamma]$ versus $\Gamma$.

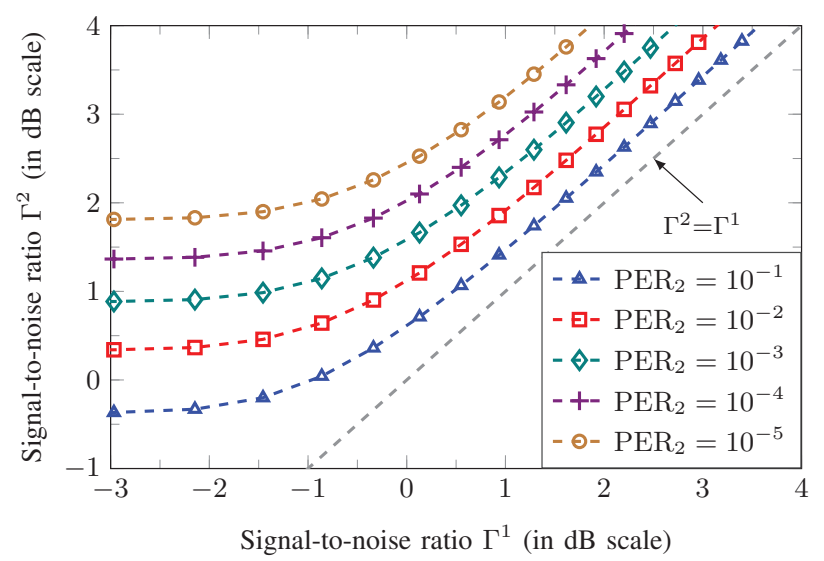

Fig. 2. Contour of the bivariate PER for the DVB-RCS turbo code [35] in the $\Gamma^{1} \times \Gamma^{2}$ plane: $\operatorname{PER}_{2}\left[\Gamma^{1}, \Gamma^{2}\right]=\operatorname{PER}\left[\Gamma^{2}\right] / \operatorname{PER}\left[\Gamma^{1}\right]$.

PER curves are drawn in Fig. 1. For the computation of that of the DVB-RCS, we estimate PER for some $\Gamma$ points (circle markers in Fig. 1), after which we interpolate and smoothen the resulting trace, as shown in a dashed line. We draw, by way of example, the bivariate PER only for the DVB-RCS in Fig. 2. Moreover, the channel power gain profile $h\left(t_{1}\right)$ is computed from the quantile function of a log-normal distribution with zero mean and deviation $3 \mathrm{~dB}$; for practical purposes, channel power gains with tail probability lower than $10^{-3}$ are discarded. We adopt the same gateway operation as in [27]: quasi-synchronous access and the decorrelation factor $\theta=1$. The noise term $N_{0}$ is set to 1 . For system optimization, we solve the problems stated in Section $\mathrm{V}$ by dividing the continuous interval $0 \leq t_{1} \leq 1$ in $r=1000$ subintervals as $t_{1}^{i}=i / r$. Throughout this study, we investigate the hitherto unexplored benefits of the two-iteration SIC (2-SIC) relative to the one-iteration SIC (1-SIC) when all users are active (i.e. $t_{*}=1$ ), and the reliability constraint is set to $c=10^{-4}$.

Our first study investigates the impact that the steepness of the PER curve of the employed coding scheme produces over energy expenditure. To that aim, we consider the theoretical coding schemes described before, and we sweep different

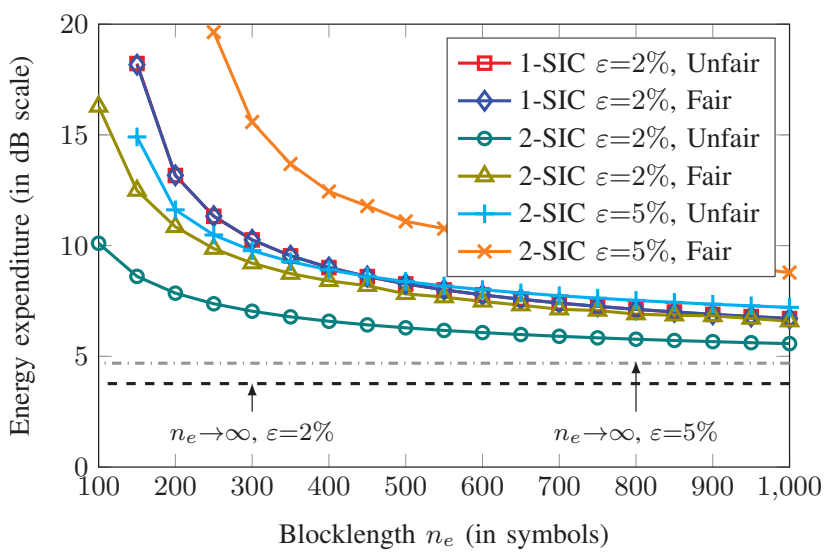

Fig. 3. Energy expenditure versus blocklength at $\alpha=3.50$.

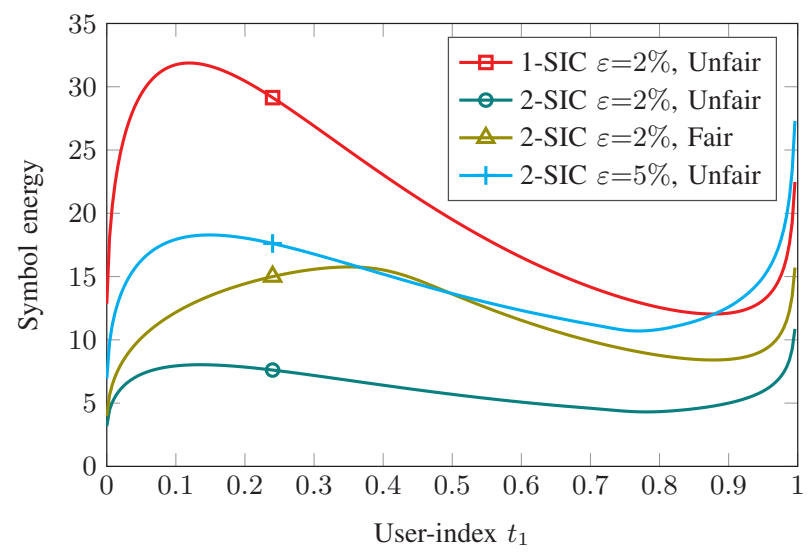

Fig. 4. Transmitted energy profile $E_{\mathrm{x}}\left(t_{1}\right)$ at $\alpha=3.50$.

blocklengths $n_{e}$ maintaining the coding rate $R=2 / 3$. This produces, as shown in Fig. 1, a number of PER curves from less to more abrupt as $n_{e}$ increases. We draw in Fig. 3 the obtained results. As shown, energy expenditure decreases with $n_{e}$ since powerful coding schemes can be designed at higher $n_{e}$, and thus, the superimposed reliability constraint is attainable with less energy expenditure. Fair allocation attains practically the optimal performance for the 1-SIC only if $\varepsilon$ is sufficiently small such that, the optimal allocation profiles with fair and unfair allocation practically coincide: the optimal allocation is uniform in SINR under fair allocation, and practically uniform under the average reliability constraint. Contrarily, fair allocation substantially degrades the performance of the 2-SIC receiver, since the optimal profile achieved under an average reliability constraint differs notably from the constant SINR profile allocated with the fair criterion. The higher the $\varepsilon$, the more highlighted the difference. Remarkably, iterative SIC benefits from unbalanced reliabilities for all users, and exploits system performance when it operates together with cancellation systems having a high uncanceled energy factor. In all cases, energy expenditure is still far from that obtained using a capacity-achieving coding scheme. 


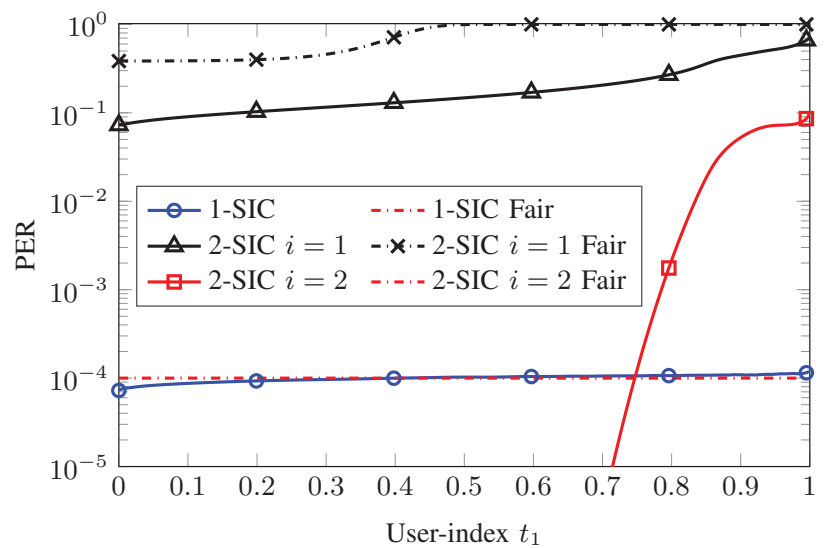

Fig. 5. User-PER profile at $\alpha=3.50, n_{e}=200$ and $\varepsilon=0.02$. The results in each iteration of the 2-SIC are labeled as $i=\{1,2\}$.

Some examples of the designed allocation profiles are depicted in Fig. 4. As corroborated by other works, twoiteration SIC allows for a high reduction of energy unbalance. Remarkably, the transmitted energy profiles tend to be uniform the more iterations are performed, except for the energy allocated to the first and last users.

As shown in Fig. 5 for the 1-SIC under unfair allocation, user-PERs are concentrated around $c=10^{-4}$ with the first users allocated to higher SINRs in detriment of the SINRs for the last users; this protects the last users from the increasing interference from unsuccessfully decoded and imperfectly canceled users. Rather, a completely different behavior is experienced for the 2-SIC. In the first SIC iteration $(i=1)$, users are allocated to low SINRs so that the receiver operates to remove a high percentage of interference awaiting for the next iteration $(i=2)$, which exploits the available decoding performance by providing almost reliable communication to a high percentage of users. The worst behavior only affects the very last users, which experience high PER values. As we increase the blocklength, the former affects fewer users. For the case depicted in Fig. 5, the $80 \%$ of users are removed in the first iteration whereas the second iteration ends with the $99.99 \%$ of users canceled.

Our second study analyzes energy expenditure versus traffic load $\alpha$ in Fig 6. As shown, energy expenditure is increasing in $\alpha$ since more energy is required to counteract multiple access interference in order to maintain the same reliability constraint. Energy expenditure for the 2-SIC is much lower at low blocklengths, specially so, at high activity loads. For increasing blocklengths or when the PER curve of the employed coding scheme is very abrupt, 1-SIC and 2-SIC almost attain the same performance. Yet, we have found the results still far from energy expenditure in the infinite blocklength regime, where, as proved in Section V-A, users attain the same SINRs regardless of the traffic load $\alpha$. Remarkably, the system does not admit infinite traffic load even if we accept energy expenditure tending to infinity; this behavior is due exclusively to imperfect cancellation. A SIC receiver with perfect cancellation $\varepsilon[\Gamma]=0$ admits an infinite load if we

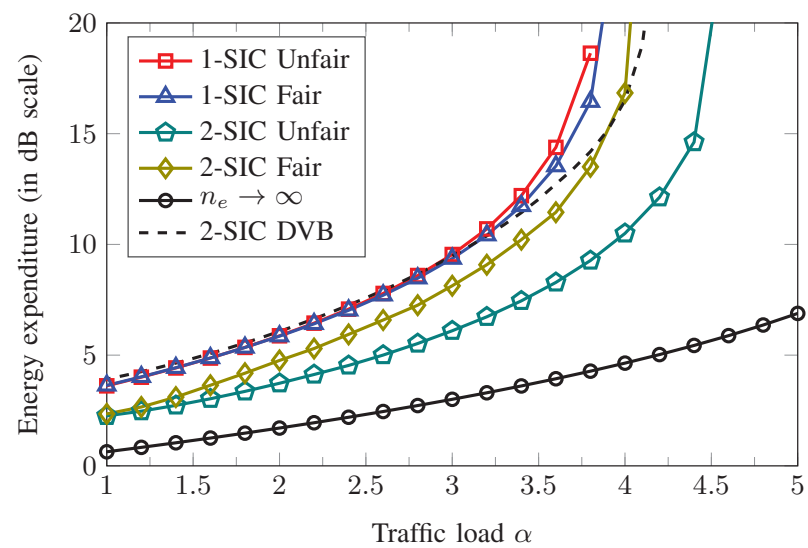

Fig. 6. Energy expenditure versus traffic load $\alpha$. The blocklength for the theoretical encoders is $n_{e}=200$ symbols, and the coding scheme from DVB-RCS operates with 192 symbols.

also spend an increasingly high amount of energy. Recall that, from a theoretic point of view, $\varepsilon$ appears inside the logarithm in the achievability region (43) for infinite-length encoders; the same behavior is demonstrated for short-length encoders at simulation level.

\section{B. Validation of the Theoretical Study}

We validate the previous theoretical work with results for an iterative SIC low-level implementation. Firstly, in the adopted satellite setting we assume each user's channel varies sufficiently slowly, with negligible changes in the received symbol energy distribution during a sufficiently long period such that, the user-PERs (34) can also be obtained empirically. Our simulations average $10^{5}$ Monte Carlo runs. The uplink channel power gains are taken from a unit-mean log-normal distribution with deviation 3dB. Secondly, we adopt a wellknown physical layer for users: the same turbo code from DVB with QPSK modulation [35] and the square-root-raisedcosine pulse with roll-off 0.22 for the chip pulse. The chip rate is fixed at 3.84 Mchips/s.

For the satellite's traveling-wave tube amplifier (TWTA), we evaluate two cases: (i) a nonlinear TWTA with inputoutput gain characteristic obtained from Saleh's model [36]; and (ii) an ideal TWTA with a two-piecewise linear gain characteristic. The input saturation power is $-84 \mathrm{dBm}$, and the output saturation power compensates for downlink path loss.

We have followed the system optimization described before with $r=1000$ intervals. We validate the theoretical findings for a low-level implementation of iterative SIC that operates at chip-level. The adopted SIC implementation, first described in Sections II-B and II-C, is summarized below to evidence the operations carried out ideally or more practically: packets are ranked according to the theoretical order of received symbol energies; the first SIC iteration processes all users; the strongest user is demodulated first using the known preamble and spreading sequence; channel decoding operates over the detected symbol sequence under 20 max-log-map iterations; ideal CRC is used to determine packet error; complex amplitude is estimated using the entire preamble plus reconstructed 


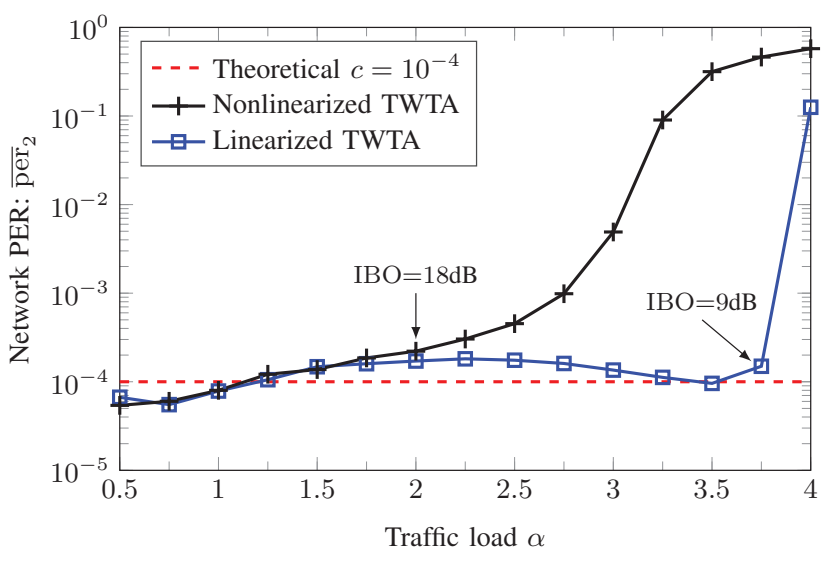

Fig. 7. Network PER (34) versus traffic load $\alpha=K / N$ with $N=64$. IBO is the input power back-off.

payload (we consider the carrier frequency known to the receiver), after which cancellation is produced. This process is repeated for other users in the decoding order established previously. For the second SIC iteration, we proceed as in the first SIC iteration but only for the users yet uncanceled.

We elaborate on the accuracy of the asymptotic study for a finite number of users. We depict in Fig. 7 the theoretical and empirical network PER versus the load $\alpha$ for the same spreading gain $N$, which causes a higher number of users $\alpha N$ as $\alpha$ increases. The simulation considers that channel power gains are uniformly sampled from the profile $h\left(t_{1}\right)$ as $t_{1}^{k}=k / K$ for $k=1, \ldots, K$, and thus, the transmitted symbol energies are interpolated from the optimized symbol energy distribution $E_{\mathrm{x}}\left(t_{1}\right)$. Recall that, the power received at the satellite's input increases in $\alpha$ because users transmit higher energy, and thus, the amplifier's operating point approaches saturation as $\alpha$ increases. Theoretically, the network PER is invariant in $\alpha$ since it is constrained to $c=10^{-4}$. The empirical simulations practically match the theoretical behavior when the operating point is far enough from saturation. The depicted results have shown the benefit of linearizing the TWTA: more users are supported without sacrificing performance. The best reported results have shown that the system model analysis of iterative SIC under linear amplification can be applied for operating points $9 \mathrm{~dB}$ below saturation. The reason is due to the power distribution at the TWTA's input; even if the operating point is far from saturation, such a distribution has a long tail, which is nonlinearly amplified, thus heavily degrading system performance.

In Fig. 8, we depict the user-PER at the traffic load $\alpha=3.00$ for $K=192$ users. Our analysis is twofold: firstly, when channel power gains are sampled uniformly from $h\left(t_{1}\right)$; and secondly, when they are sampled randomly from $h\left(t_{1}\right)$, which causes, at every simulation, slightly different distributions for transmitted symbol energies depending on the number of users $\alpha N$. As shown, the empirical user-PERs show similarities in terms of trend but with some differences with respect to the theoretical prediction. The reason is that the true behavior of iterative SIC is very sensitive to small changes in the received distributions. The user-asymptotic approximation to a finite

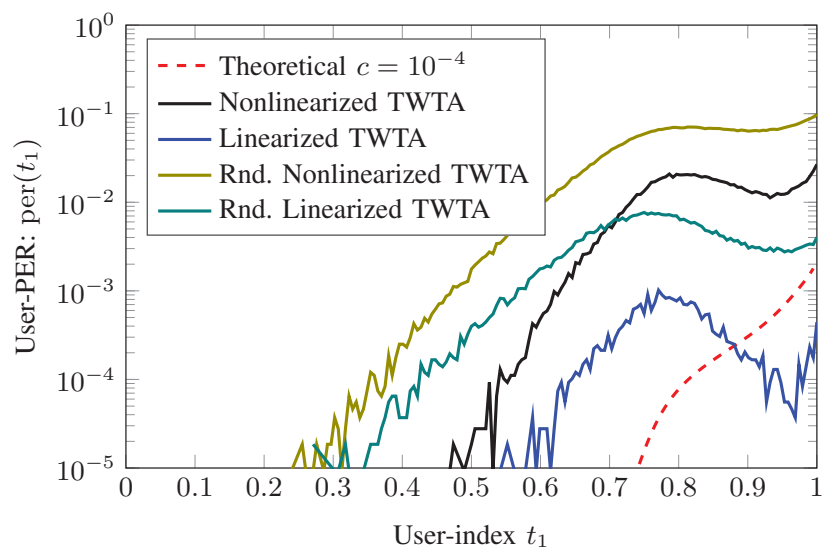

Fig. 8. User-PER profile after two SIC iterations (35) at $\alpha=K / N=3.00$ with $N=64$

number of users, and the approximation of the true bivariate PER by (18) entail small mismatches that, in combination with each stage of SIC, accumulate errors that are increased as the SIC receiver advances. Remarkably, SIC's performance is strongly dependent on the received symbol energy distribution, which varies sufficiently if the number of users $\alpha N$ is low.

\section{CONCLUSIONS}

We have addressed the energy allocation design from an energy efficiency perspective for a satellite setting with massive connectivity and a gateway employing a interference cancellation strategy. We have solved the shared access problem by allowing the users to employ a simplified physical layer consisting of the same coding scheme followed by non-orthogonal multiple access; at the gateway, multiple access interference has been counteracted by iterative SIC. In this respect, we have investigated the asymptotically optimal energy allocation design for the two first iterations, as they mainly contribute to mitigating interference. We have modeled channel decoding using multivariate PER functions in each iteration. With all this, we have tackled the allocation problem so as to minimize energy expenditure while guaranteeing either network or user reliability constraints. Our main results, obtained via simulations, have shown that iterative SIC outperforms the classical non-iterative SIC, specially in the very short packet regime under demanding service requirements or in a high activity situation. Moreover, with a network reliability constraint, twoiteration SIC provides almost reliable communication to most users except to the very last ones. Our contribution has overcome the deficiencies in the previous works, by adopting a low-level system implementation that validates our study for some hundreds of users when the operating point of the satellite amplifier is sufficiently far from saturation.

\section{APPENDIX 1}

We derive in this appendix the variational derivatives corresponding to the integrands of the profit and the reliability constraint with a one-iteration SIC. To shorten notation, functions of the user-variable $t_{1}$ are denoted in boldface. 
Firstly, with regard to the energy expenditure profit

$$
\bar{E}=\int_{0}^{t_{*}} \boldsymbol{E}_{\mathbf{x}} \mathrm{d} t_{1}
$$

the computation of its variational derivative is straightforward from the previous functional:

$$
\frac{\delta \boldsymbol{E}_{\mathbf{x}}}{\delta \boldsymbol{E}_{\mathbf{x}}}=1 .
$$

Secondly, with regard to the constraint

$$
C=\int_{0}^{t_{*}} \operatorname{per} \mathrm{d} t_{1}=\int_{0}^{t_{*}} \operatorname{PER}\left[\boldsymbol{\Gamma}^{\mathbf{1}}\right] \mathrm{d} t_{1}
$$

we compute its first variation considering variations $\boldsymbol{E}_{\mathbf{x}}+\boldsymbol{v}$ that produce, at the same time, variations $\phi_{1}$ over the noise plus interference profile $N_{t}^{1}$, as $N_{t}^{1}+\phi_{1}$. We have

$$
\delta C=\int_{0}^{t_{*}} \operatorname{PER}^{\prime}\left[\boldsymbol{\Gamma}^{\mathbf{1}}\right]\left(\frac{\boldsymbol{h}}{\boldsymbol{N}_{\boldsymbol{t}}^{\mathbf{1}}} \boldsymbol{v}-\boldsymbol{\Gamma}^{\mathbf{1}} \frac{\phi_{\mathbf{1}}}{\boldsymbol{N}_{\boldsymbol{t}}^{\mathbf{1}}}\right) \mathrm{d} t_{1}
$$

with $\phi_{1} / N_{t}^{1}$ computed by taking variations over (22), as

$$
\frac{\phi_{\mathbf{1}}}{\boldsymbol{N}_{\boldsymbol{t}}^{\mathbf{1}}}=\frac{\phi_{\mathbf{1}}(0)}{\boldsymbol{N}_{\boldsymbol{t}}^{\mathbf{1}}(0)}-\alpha \int_{0}^{t_{1}} \Phi^{\prime}\left[\boldsymbol{\Gamma}^{\mathbf{1}}\right]\left(\frac{\boldsymbol{h}}{\boldsymbol{N}_{\boldsymbol{t}}^{\mathbf{1}}} \boldsymbol{v}-\boldsymbol{\Gamma}^{\mathbf{1}} \frac{\phi_{\mathbf{1}}}{\boldsymbol{N}_{\boldsymbol{t}}^{\mathbf{1}}}\right) \mathrm{d} \tau .
$$

The initial value is $\boldsymbol{\phi}_{\mathbf{1}}(0)=\alpha \theta \int_{0}^{t_{*}} \boldsymbol{h} \boldsymbol{v} \mathrm{d} t_{1}$. To isolate $\boldsymbol{\phi} / \boldsymbol{N}_{\boldsymbol{t}}^{\mathbf{1}}$, we compute its derivative

$$
\left(\frac{\phi_{1}}{\boldsymbol{N}_{t}^{1}}\right)^{\prime}=-\alpha \Phi^{\prime}\left[\boldsymbol{\Gamma}^{1}\right]\left(\frac{\boldsymbol{h}}{\boldsymbol{N}_{t}^{1}} v-\Gamma^{1} \frac{\phi_{1}}{\boldsymbol{N}_{t}^{1}}\right),
$$

which constitutes a first-order differential equation that we solve using the integrating factor method. Then, under $\boldsymbol{a}_{\mathbf{1}} \triangleq$ $-\alpha \Phi^{\prime}\left[\boldsymbol{\Gamma}^{\mathbf{1}}\right] \boldsymbol{h} / \boldsymbol{N}_{\boldsymbol{t}}^{\mathbf{1}}$ and $\boldsymbol{b}_{\mathbf{1}} \triangleq \exp \left(\alpha \int_{0}^{t_{1}} \Phi^{\prime}\left[\boldsymbol{\Gamma}^{\mathbf{1}}\right] \boldsymbol{\Gamma}^{\mathbf{1}} \mathrm{d} \tau\right)$, we get

$$
\frac{\phi_{1}}{N_{t}^{1}}=b_{1} \int_{0}^{t_{1}} \frac{a_{1}}{b_{1}} v \mathrm{~d} \tau+\frac{\phi_{1}(0)}{N_{t}^{1}(0)} b_{1} .
$$

The previous result is substituted in (55), after which, by integrating by parts and by rearranging terms we can obtain an integral linear in $\boldsymbol{v}$

$$
\delta C=\int_{0}^{t_{*}}\left(\frac{\delta \text { per }}{\delta \boldsymbol{E}_{\mathbf{x}}}\right) \boldsymbol{v} \mathrm{d} t_{1}
$$

whose integrand corresponds, exactly, with the sought variational derivative:

$$
\begin{aligned}
\frac{\delta \text { per }}{\delta \boldsymbol{E}_{\mathrm{x}}} & =\operatorname{PER}^{\prime}\left[\boldsymbol{\Gamma}^{\mathbf{1}}\right] \frac{\boldsymbol{h}}{\boldsymbol{N}_{\boldsymbol{t}}^{\mathbf{1}}}-\frac{\boldsymbol{a}_{\mathbf{1}}}{\boldsymbol{b}_{\mathbf{1}}} \boldsymbol{c}_{\mathbf{1}}-\boldsymbol{h} \frac{\alpha \theta \boldsymbol{c}_{\mathbf{1}}(0)}{\boldsymbol{N}_{\boldsymbol{t}}^{\mathbf{1}}(0)}, \\
\boldsymbol{c}_{\mathbf{1}} & \triangleq \int_{t_{1}}^{t_{*}} \boldsymbol{b}_{\mathbf{1}} \mathrm{PER}^{\prime}\left[\boldsymbol{\Gamma}^{\mathbf{1}}\right] \boldsymbol{\Gamma}^{\mathbf{1}} \mathrm{d} \tau .
\end{aligned}
$$

\section{APPENDIX 2}

We extend, in this appendix, the procedure followed in Appendix I to obtain the variational derivatives when a twoiteration SIC operates. Specifically, energy expenditure shows the same form presented before whereas the first variation of the constraint

$$
C=\int_{0}^{t_{*}} \operatorname{per} \mathrm{d} t_{1}=\int_{0}^{t_{1}} \operatorname{PER}\left[\boldsymbol{\Gamma}^{2}\right] \mathrm{d} t_{1}
$$

shows a substantially different form:

$$
\delta C=\int_{0}^{t_{*}} \operatorname{PER}^{\prime}\left[\boldsymbol{\Gamma}^{\mathbf{2}}\right]\left(\frac{\boldsymbol{h}}{\boldsymbol{N}_{\boldsymbol{t}}^{\mathbf{2}}} \boldsymbol{v}-\boldsymbol{\Gamma}^{\mathbf{2}} \frac{\phi_{\mathbf{2}}}{\boldsymbol{N}_{\boldsymbol{t}}^{\mathbf{2}}}\right) \mathrm{d} t_{1}
$$

with

$$
\begin{aligned}
\frac{\phi_{\mathbf{2}}}{\boldsymbol{N}_{\boldsymbol{t}}^{\mathbf{2}}} & =\frac{\phi_{\mathbf{1}}\left(t_{*}\right)}{\boldsymbol{N}_{\boldsymbol{t}}^{\mathbf{1}}\left(t_{*}\right)}-\alpha \int_{0}^{t_{1}} \Psi_{\Gamma^{1}}\left[\boldsymbol{\Gamma}^{\mathbf{1}}\right]\left(\frac{\boldsymbol{h}}{\boldsymbol{N}_{\boldsymbol{t}}^{\mathbf{1}}} \boldsymbol{v}-\boldsymbol{\Gamma}^{\mathbf{1}} \frac{\phi_{\mathbf{1}}}{\boldsymbol{N}_{\boldsymbol{t}}^{\mathbf{1}}}\right) \mathrm{d} \tau \\
& -\alpha \int_{0}^{t_{1}} \Psi_{\Gamma^{2}}\left[\boldsymbol{\Gamma}^{\mathbf{1}}, \boldsymbol{\Gamma}^{\mathbf{2}}\right]\left(\frac{\boldsymbol{h}}{\boldsymbol{N}_{\boldsymbol{t}}^{\mathbf{2}}} \boldsymbol{v}-\boldsymbol{\Gamma}^{\mathbf{2}} \frac{\phi_{\mathbf{2}}}{\boldsymbol{N}_{\boldsymbol{t}}^{\mathbf{2}}}\right) \mathrm{d} \tau
\end{aligned}
$$

The differential equation

$$
\left(\frac{\phi_{\mathbf{2}}}{\boldsymbol{N}_{\boldsymbol{t}}^{2}}\right)^{\prime}=\boldsymbol{a}_{2}+\alpha \Psi_{\Gamma^{2}}\left[\boldsymbol{\Gamma}^{\mathbf{1}}, \boldsymbol{\Gamma}^{2}\right] \frac{\phi_{2}}{\boldsymbol{N}_{\boldsymbol{t}}^{2}}
$$

with

$$
\begin{aligned}
\boldsymbol{a}_{\mathbf{2}} & \triangleq-\alpha\left(\frac{\Psi_{\Gamma^{1}}\left[\boldsymbol{\Gamma}^{\mathbf{1}}, \boldsymbol{\Gamma}^{2}\right]}{\boldsymbol{N}_{\boldsymbol{t}}^{\mathbf{1}}}+\frac{\Psi_{\Gamma^{2}}\left[\boldsymbol{\Gamma}^{\mathbf{1}}, \boldsymbol{\Gamma}^{2}\right]}{\boldsymbol{N}_{\boldsymbol{t}}^{2}}\right) \boldsymbol{h} \boldsymbol{v} \\
& +\alpha \Psi_{\Gamma^{1}}\left[\boldsymbol{\Gamma}^{\mathbf{1}}, \boldsymbol{\Gamma}^{\mathbf{2}}\right] \boldsymbol{\Gamma}^{\mathbf{1}} \frac{\boldsymbol{\phi}_{\mathbf{1}}}{\boldsymbol{N}_{\boldsymbol{t}}^{\mathbf{1}}}
\end{aligned}
$$

can be solved by means of the integrating factor method. This gives, under $\boldsymbol{b}_{\mathbf{2}} \triangleq \exp \left(\alpha \int_{0}^{t_{1}} \Psi_{\Gamma^{2}}\left[\boldsymbol{\Gamma}^{\mathbf{1}}, \boldsymbol{\Gamma}^{\mathbf{2}}\right] \boldsymbol{\Gamma}^{\mathbf{2}} \mathrm{d} \tau\right)$,

$$
\frac{\phi_{\mathbf{2}}}{\boldsymbol{N}_{\boldsymbol{t}}^{\mathbf{2}}}=\boldsymbol{b}_{\mathbf{2}} \int_{0}^{t_{1}} \frac{\boldsymbol{a}_{\mathbf{2}}}{\boldsymbol{b}_{\mathbf{2}}} \boldsymbol{v} \mathrm{d} \tau+\frac{\phi_{\mathbf{1}}\left(t_{*}\right)}{\boldsymbol{N}_{\boldsymbol{t}}^{\mathbf{1}}\left(t_{*}\right)} \boldsymbol{b}_{\mathbf{2}}
$$

Now, substituting (58) particularized at $t_{1}=t_{*}$ in (67) and the resulting (67) in (63), we get, after some straightforward manipulations, the following variational derivative

$$
\begin{aligned}
\frac{\delta \text { per }}{\delta \boldsymbol{E}_{\mathrm{x}}} & =\operatorname{PER}^{\prime}\left[\boldsymbol{\Gamma}^{\mathbf{2}}\right] \frac{\boldsymbol{h}}{\boldsymbol{N}_{\boldsymbol{t}}^{\mathbf{2}}}-\frac{\boldsymbol{a}_{\mathbf{1}}}{\boldsymbol{b}_{\mathbf{1}}} \boldsymbol{e}_{\mathbf{2}}-\beta \boldsymbol{c}_{\mathbf{2}}(0)-\lambda \boldsymbol{e}_{2}(0) \\
& +\alpha \frac{\boldsymbol{c}_{\mathbf{2}}}{\boldsymbol{b}_{\mathbf{2}}} \boldsymbol{h}\left(\frac{\Psi_{\Gamma^{1}}\left[\boldsymbol{\Gamma}^{\mathbf{1}}, \boldsymbol{\Gamma}^{\mathbf{2}}\right]}{\boldsymbol{N}_{\boldsymbol{t}}^{\mathbf{1}}}+\frac{\Psi_{\Gamma^{2}}\left[\boldsymbol{\Gamma}^{\mathbf{1}}, \boldsymbol{\Gamma}^{\mathbf{2}}\right]}{\boldsymbol{N}_{\boldsymbol{t}}^{\mathbf{2}}}\right)
\end{aligned}
$$

with

$$
\begin{aligned}
& \boldsymbol{c}_{\mathbf{2}} \triangleq \int_{t_{1}}^{t_{*}} \operatorname{PER}^{\prime}\left[\boldsymbol{\Gamma}^{2}\right] \boldsymbol{\Gamma}^{2} \boldsymbol{b}_{\mathbf{2}} \mathrm{d} \tau, \\
& \boldsymbol{e}_{\mathbf{2}} \triangleq \alpha \int_{t_{1}}^{t_{*}} \frac{\boldsymbol{c}_{\mathbf{2}}}{\boldsymbol{b}_{\mathbf{2}}} \Psi_{\Gamma^{1}}\left[\boldsymbol{\Gamma}^{\mathbf{1}}, \boldsymbol{\Gamma}^{\mathbf{2}}\right] \boldsymbol{\Gamma}^{\mathbf{1}} \boldsymbol{b}_{\mathbf{1}} \mathrm{d} \tau .
\end{aligned}
$$

\section{REFERENCES}

[1] A. Yadav and O. A. Dobre, "All technologies work together for good: A glance at future mobile networks," IEEE Wireless Commun., vol. 25, no. 4, pp. 10-16, 2018.

[2] R. De Gaudenzi, O. Del Río Herrero, S. Cioni, and A. Mengali, Random Access Versus Multiple Access. Cham: Springer International Publishing, 2019, pp. 535-584.

[3] J. A. Fraire, J. M. Finochietto, and S. C. Burleigh, Delay Tolerant Satellite Networks. Artech House, 2017.

[4] S. Cioni, R. De Gaudenzi, O. Del Río Herrero, and N. Girault, "On the satellite role in the era of $5 \mathrm{G}$ massive machine type communications," IEEE Netw., vol. 32, no. 5, pp. 54-61, 2018.

[5] J. A. Fraire, S. Céspedes, and N. Accettura, "Direct-to-satellite IoT a survey of the state of the art and future research perspectives," in ADHOC-NOW 2019: Ad-Hoc, Mobile, and Wireless Networks, Luxembourg, Luxembourg, Oct. 2019, pp. 241-258.

[6] M. De Sanctis, E. Cianca, G. Araniti, I. Bisio, and R. Prasad, "Satellite communications supporting internet of remote things," IEEE Internet Things J., vol. 3, no. 1, pp. 113-123, 2016.

[7] M. Luglio, S. P. Romano, C. Roseti, and F. Zampognaro, "Service delivery models for converged satellite-terrestrial $5 \mathrm{G}$ network deployment: A satellite-assisted CDN use-case," IEEE Network, vol. 33, no. 1, pp $142-150,2019$. 
[8] N. Celandroni, E. Ferro, A. Gotta, G. Oligeri, C. Roseti, M. Luglio, I. Bisio, M. Cello, F. Davoli, A. D. Panagopoulos, M. Poulakis, S. Vassaki, T. De Cola, M. A. Marchitti, Y. F. Hu, P. Pillai, S. Verma, $\mathrm{K}$. Xu, and G. Acar, "A survey of architectures and scenarios in satellitebased wireless sensor networks: system design aspects," Int. Journal of Satellite Communications and Networking, vol. 31 , no. 1, pp. 1-38, 2013.

[9] O. Kodheli, E. Lagunas, N. Maturo, S. K. Sharma, B. Shankar, J. F. M. Montoya, J. C. M. Duncan, D. Spano, S. Chatzinotas, S. Kisseleff, J. Querol, L. Lei, T. X. Vu, and G. Goussetis, "Satellite communications in the new space era: A survey and future challenges," IEEE Commun. Surveys Tuts., vol. 23, no. 1, pp. 70-109, 2021.

[10] R. De Gaudenzi, O. Del Rio Herrero, G. Gallinaro, C. Stefano, and A. Pantelis-Daniel, "Random access schemes for satellite networks, from VSAT to M2M: a survey," Int. Journal of Satellite Communications and Networking, vol. 36, no. 1, pp. 66-107, 2018.

[11] M. B. Shahab, R. Abbas, M. Shirvanimoghaddam, and S. J. Johnson, "Grant-free non-orthogonal multiple access for IoT: A survey," IEEE Commun. Surveys Tuts., vol. 22, no. 3, pp. 1805-1838, 2020.

[12] J. Su, G. Ren, Q. Wang, and H. Zhang, "Randomly pre-coded packets based random access scheme for IoT-oriented satellite networks," IEEE Access, vol. 8, pp. 221 148-221 161, 2020.

[13] A. Mengali, R. De Gaudenzi, and P. Arapoglou, "Enhancing the physical layer of contention resolution diversity slotted ALOHA," IEEE Trans. Commun., vol. 65, no. 10, pp. 4295-4308, 2017.

[14] S. Verdú, Multiuser detection. Cambridge University Press, 1998.

[15] P. S. Rossi, K. Kansanen, R. R. Müller, and C. Rachinger, "Power randomization for iterative detection over random-access fading channels," IEEE Trans. Wireless Commun., vol. 14, no. 10, pp. 5704-5713, 2015.

[16] M. T. P. Le, G. C. Ferrante, T. Q. S. Quek, and M. Di Benedetto, "Fundamental limits of low-density spreading NOMA with fading," IEEE Trans. Wireless Commun., vol. 17, no. 7, pp. 4648-4659, 2018.

[17] M. Andrenacci, G. Mendola, F. Collard, D. Finocchiaro, and A. Recchia, "Enhanced spread spectrum aloha demodulator implementation, laboratory tests and satellite validation," Int. Journal of Satellite Communications and Networking, vol. 32, no. 6, pp. 521-533, 2014.

[18] A. Arcidiacono, D. Finocchiaro, F. Collard, S. Scalise, F. Lazaro Blasco, R. De Gaudenzi, S. Cioni, N. Alagha, and M. Andrenacci, "From S-band mobile interactive multimedia to fixed satellite interactive multimedia: making satellite interactivity affordable at Ku-band and Ka-band," Int. Journal of Satellite Communications and Networking, vol. 34, no. 4, pp. 575-601, 2016.

[19] Z. Ding, R. Schober, and H. V. Poor, "Unveiling the importance of SIC in NOMA systems - part I: State of the art and recent findings," IEEE Commun. Lett., vol. 24, no. 11, pp. 2373-2377, 2020.

[20] — "Unveiling the importance of SIC in NOMA systems-part II: New results and future directions," IEEE Commun. Lett., vol. 24, no. 11, pp. 2378-2382, 2020.

[21] D. V. Djonin and V. K. Bhargava, "Asymptotic analysis of the conventional decision feedback receiver in fading channels," IEEE Trans. Wireless Commun., vol. 2, no. 5, pp. 1066-1078, 2003.

[22] Y. Polyanskiy, H. V. Poor, and S. Verdú, "Channel coding rate in the finite blocklength regime," IEEE Trans. Info. Theory, vol. 56, no. 5, pp. 2307-2359, 2010.

[23] Y. Yu, H. Chen, Y. Li, Z. Ding, and B. Vucetic, "On the performance of non-orthogonal multiple access in short-packet communications," IEEE Commun. Lett., vol. 22, no. 3, pp. 590-593, 2018.

[24] F. Ghanami, G. A. Hodtani, B. Vucetic, and M. Shirvanimoghaddam, "Performance analysis and optimization of NOMA with HARQ for short packet communications in massive IoT," IEEE Internet Things J., vol. 8, no. 6 , pp. 4736-4748, 2021.

[25] P. Cassará, T. De Cola, and A. Gotta, "A statistical framework for performance analysis of diversity framed slotted aloha with interference cancellation," IEEE Trans. Aerosp. Electron. Syst., vol. 56, no. 6, pp. 4327-4337, 2020.

[26] J. Sala-Álvarez, F. Rey, J. Villares, and F. Molina, "Minimum PER user-energy profile for massive SIC receivers under an average energy constraint," in 2017 IEEE Int. Workshop on Signal Processing Advances in Wireless Communications, Jul. 2017, pp. 1-6.

[27] F. Molina and J. Sala-Álvarez, "Energy allocation for short-packet massive multiple access with two-iteration successive decoding," in 2020 IEEE Int. Conference on Communications, May 2020, pp. 1-6.

[28] F. Collard and R. De Gaudenzi, "On the optimum packet power distribution for spread ALOHA packet detectors with iterative successive interference cancelation," IEEE Trans. on Wireless Commun., vol. 13, no. 12, pp. 6783-6794, 2014.
[29] F. Molina and J. Sala-Álvarez, "Average PER performance metrics of iterative successive interference cancellation," IEEE Wireless Commun. Lett., vol. 9, no. 1, pp. 74-77, 2020.

[30] S. Alvi, S. Durrani, and X. Zhou, "Enhancing CRDSA with transmit power diversity for machine-type communication," IEEE Trans. Veh. Technol., vol. 67, no. 8, pp. 7790-7794, 2018.

[31] Y. Xu, C. Shen, T. Chang, S. Lin, Y. Zhao, and G. Zhu, "Transmission energy minimization for heterogeneous low-latency NOMA downlink," IEEE Trans. Wireless Commun., vol. 19, no. 2, pp. 1054-1069, 2020.

[32] J. Qiu, D. Abe, T. Antonsen, B. Danly, B. Levush, and R. Myers, "Linearizability of TWTAs using predistortion techniques," IEEE Trans. Electron Devices, vol. 52, no. 5, pp. 718-727, 2005.

[33] A. Katz, R. Gray, and R. Dorval, "Wide/multiband linearization of TWTAs using predistortion," IEEE Trans. Electron Devices, vol. 56, no. 5, pp. 959-964, 2009.

[34] R. Buehrer, "Equal BER performance in linear successive interference cancellation for CDMA systems," IEEE Trans. Commun., vol. 49, no. 7, pp. 1250-1258, 2001.

[35] EN ETSI, “301 790 v1.5.1 (2009-05): Digital video broadcasting (DVB); interaction channel for satellite distribution systems," 2009.

[36] A. Saleh, "Frequency-independent and frequency-dependent nonlinear models of TWT amplifiers," IEEE Trans. Commun., vol. 29, no. 11, pp. $1715-1720,1981$.

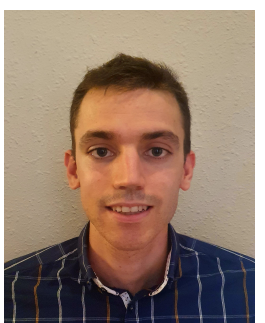

Francesc Molina (S'17) was born in Barcelona, Spain, in 1993. He received the Telecommunications Engineering degree and the MSc degree from Universitat Politècnica de Catalunya, Barcelona, Spain, in 2015 and 2017, respectively. In 2016, he joined the Signal Processing and Communications (SP$\mathrm{COM}$ ) group at Universitat Politècnica de Catalunya as assistant researcher, where he is currently pursuing the $\mathrm{PhD}$ degree. In 2018, he held the Grant for the recruitment of new research staff (FI-2018) from the Catalan administration AGAUR to complete the $\mathrm{PhD}$ degree. His research interests span the fields of wireless communication and information theory. His research topic is massive multiple access.

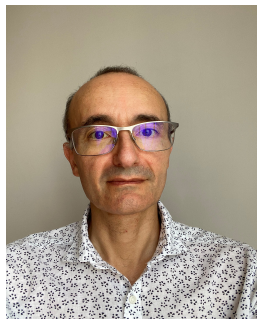

Josep Sala-Álvarez (S'86-SM'11) was born in Barcelona, Spain, in 1967. He received his M.Sc. and Ph.D. degrees in telecommunications engineering from the Technical University of Catalonia (UPC), Barcelona, Spain, in 1991 and 1995, respectively. During 1992, he worked at the European Space Operations Centre, European Space Agency (ESA), Darmstadt, Germany, in the area of software engineering for telemetry processing. From 1993 to late 1994, he held a PhD grant from the Catalan Administration at the Department of Signal Theory and Communications, UPC, which he joined as an Assistant Professor in 1994. He was promoted to Associate Professor in 1997. He has participated in over forty projects, both in space communications for ESA and in wireless communications for industry and institutions at the national/European level. His current research interests include the field of signal processing, communications, and information theory. He received the IEEE Signal Processing Society Best (Senior) Paper Award (2003), the International Symposium on Turbo-Codes and Applications (ISTC 2003) Best Poster Paper Award, and the Best Ph.D. Thesis in Telecommunications National Award (Spain, 1995). He has published over eighty journal and conference papers. 